\title{
Parliamentary Voting Procedures: Agenda Control, Manipulation, and Uncertainty
}

\author{
Robert Bredereck \\ Institut für Softwaretechnik und Theoretische Informatik \\ TU Berlin, Germany \\ Jiehua Chen \\ Dept. Industrial Engineering and Management \\ Ben-Gurion University of the Negev, Israel
}

\section{Rolf Niedermeier}

Toby Walsh

Institut für Softwaretechnik und Theoretische Informatik

TU Berlin, Germany
ROBERT.BREDERECK@TU-BERLIN.DE

JIEHUA.CHEN2@GMAIL.COM

ROLF.NIEDERMEIER@TU-BERLIN.DE

TOBY.WALSH@DATA61.CSIRO.AU

\begin{abstract}
We study computational problems for two popular parliamentary voting procedures: the amendment procedure and the successive procedure. They work in multiple stages where the result of each stage may influence the result of the next stage. Both procedures proceed according to a given linear order of the alternatives, an agenda. We obtain the following results for both voting procedures: On the one hand, deciding whether one can make a specific alternative win by reporting insincere preferences by the fewest number of voters, the COALITIONAL MANiPUlation problem, or whether there is a suitable ordering of the agenda, the Agenda Control problem, takes polynomial time. On the other hand, our experimental studies with real-world data indicate that most preference profiles cannot be manipulated by only few voters and a successful agenda control is typically impossible. If the voters' preferences are incomplete, then deciding whether an alternative can possibly win is NP-hard for both procedures. Whilst deciding whether an alternative necessarily wins is coNP-hard for the amendment procedure, it is polynomial-time solvable for the successive procedure.
\end{abstract}

\section{Introduction}

Two prominent voting procedures are used in many parliamentary chambers to amend and decide upon new legislation: the successive procedure and the amendment procedure (Black, 1958; Farquharson, 1969; Miller, 1977; Apesteguia, Ballester, \& Masatlioglu, 2014). Both are sequential (or multi-stage) voting procedures: the alternatives are ordered (thus forming an agenda) and they are considered one by one, making a binary decision based on majority voting in each step. In a nutshell, in each step, the successive procedure considers the current alternative and decides whether to accept it (in which case the procedure stops and the winner is determined) or to reject it and the procedure then continues with the remaining alternatives in the given order. The amendment procedure in each step jointly considers two 
current alternatives and decides by majority voting which one of the two is eliminated - the remaining one then will be confronted with the next alternative on the agenda.

There are many reasons to study the properties of parliamentary voting procedures, and, especially, to consider computational questions. First, parliamentary voting procedures are used very frequently in practice. For example, the recent $112^{\text {th }}$ Congress of the US Senate and House of Representatives had 1030 votes to amend and approve bills (GovTrack, 20112012). This does not take into account the hundreds of committees that also amended and voted on these bills. As a second example, there were 351 divisions within the UK Houses of Lords and Commons in 2013 to amend or approve bills. Both countries use the amendment procedure to make parliamentary decisions (Rasch, 2000).

Second, parliamentary voting procedures are used to make some of the most important decisions in society. We decide to reduce carbon emissions, provide universal health care, or raise taxes based on the outcome of such voting procedures. When rallying support for new legislation, it is vital to know what amendments (alternatives) can and cannot be passed. Fortunately, we have excellent historical voting records for parliamentary chambers. We can therefore make high quality predictions about how sincere or "sophisticated/strategic" voters will vote.

Third, Enelow and Koehler (1980, p.2) examined several votes in the US House of Representatives in 1977 and gave evidence that parliamentary voting may be strategic:

"Thus, it is shown that sophisticated voting does occur in Congress and in fact is encouraged by the way amendments are used in the legislative process.

It should not come as a surprise to congressional scholars that congressmen do not always vote sincerely."

Fourth, there is both theoretical and empirical evidence that the final outcome critically depends on the order in which amendments (alternatives) are presented. For example, Ordeshook and Schwartz (1987, p. 1) remark that

\section{"... legislative decisions are at the mercy of elites who control agendas."}

It is therefore interesting to ask whether, for example, computational complexity is a barrier to the control of the agenda (see Section 3 for more information on this question) or to strategic voting when such parliamentary voting procedures are used (see Section 4 for more information on this question). The former refers to the AGEndA Control problem while the latter to the Coalitional Manipulation problem. It is also interesting to ask if we can efficiently compute whether a particular amendment (alternative) can possibly (or necessarily) pass despite uncertainty in the votes or the agenda. This refers to the Possible Winner (or Necessary Winner) problem. We assume that we know each voter's preference order over the alternatives, which is a linear order in the first two types of problems and is a partial order in the last type of problems. This assumption is standard within computational social choice, and we can approximate how the voters would intent to vote from pre-elections. We provide one of the first computational studies of Agenda Control, Coalitional Manipulation, and Possible/Necessary Winner giving both theoretical and empirical results. 


\subsection{Related Work}

There are many studies in the economic and political sciences literature on parliamentary voting procedures, starting with the work of Black (1958) and Farquharson (1969), concerning "insincere" or "sophisticated" or "strategic" voting (Miller, 1977; Enelow \& Koehler, 1980; McKelvey \& Niemi, 1978; Shepsle \& Weingast, 1982; Banks, 1985; Moulin, 1986; Ordeshook \& Schwartz, 1987). Apesteguia et al. (2014) characterize both the amendment and the successive procedures from an axiomatic perspective.

Miller (1977) studied which alternatives can become a winner by altering the agenda, the Agenda Control problem. In particular, he showed through Propositions 1 and 2 in his paper (Miller, 1977) that if each voter votes sincerely, then an alternative can become an amendment winner under some agenda if and only if it belongs to the Condorcet set (also known as top cycle, see Nurmi, 2005); a Condorcet set is the smallest set of alternatives that beats every other alternative outside this set. We extend this result by a constructive proof. For the successive procedure, however, Miller (1977) only showed that every alternative from the Condorcet set can win. Barberà and Gerber (2017) followed Miller's research of characterizing the set of alternatives that may become an amendment (or a successive) winner by controlling the agenda. However, they assumed that the voters are voting sophisticatedly, that is, they may deviate from their true preferences if the outcome may be more preferable for them. Rasch (2014) empirically examined the behavior of voters in the Norwegian parliament, where the successive procedure is used. He reported that successful insincere voting, where voters may vote differently from their true preferences and the outcome is better for them, is very rare.

In computational social choice, the term "electoral control" refers to the situation where an external agent tries to affect the outcome by changing the structure of an existing election. Here, to affect the outcome means for instance to make some alternative(s) win (constructive control) or lose (destructive control). The structure of the election may be changed, for example, by adding or deleting alternatives or voters. Constructive control was introduced by Bartholdi III, Tovey, and Trick (1992) and has been studied extensively. Our agenda control differs from the aforementioned electoral control since we do not change the structure of the profile. We point to a recent book chapter (Faliszewski \& Rothe, 2016) and surveys (Faliszewski, Hemaspaandra, \& Hemaspaandra, 2010; Rothe \& Schend, 2013) for an extensive discussion of (non-parliamentary) electoral control.

Using computational complexity as a barrier against manipulation, that is, affecting the voting outcome by reporting insincere preferences was initiated by Bartholdi III, Tovey, and Trick (1989). They showed that manipulating a special variant of the Copeland voting rule is NP-complete even when there is only one manipulator whereas manipulating most widely-used voting rules is polynomial-time solvable in case of a single manipulator. In case of more than one manipulator, Conitzer, Sandholm, and Lang (2007) showed that Coalitional Manipulation, where each voter is associated with a weight is already NP-complete even when the number of alternatives is a small constant; their hardness results are indeed weak NP-hardness results, not excluding polynomial-time algorithms for the case that the weights are polynomially bounded. Bartholdi III and Orlin (1991) showed that manipulating the Single Transfer Vote (STV) voting rule is NP-complete even when there is only one manipulator. STV and its many variants have been adopted in the 
parliamentary voting of many countries. They are sequential voting rules and work similarly to the successive procedure except that there is no agenda. Instead, in each step, the alternative that is ranked first by the least number of voters will be deleted from the profile. This means that if an alternative $a$ is an STV winner, then there is an agenda for which alternative $a$ becomes a successive winner. The NP-completeness result for manipulating STV is of particular interest since we provide polynomial-time algorithms for manipulating the closely related successive procedure. These two complexity results indicate that it is the agenda that makes an important difference.

Concerning uncertainty in elections (that is, each voter's preference order is only a partial order), there is some work in the political science literature (Ordeshook \& Palfrey, 1988; Jung, 1989), but there seems to be significantly more activity on the computational side. Konczak and Lang (2005) introduced the problems of Possible Winner and Necessary Winner (determination), and studied them for the Condorcet rule. Since then, these problems have been frequently studied for several other common voting rules (Walsh, 2007; Betzler, Hemmann, \& Niedermeier, 2009; Betzler \& Dorn, 2010; Baumeister \& Rothe, 2012; Hazon, Aumann, Kraus, \& Wooldridge, 2012; Aziz, Brill, Fischer, Harrenstein, Lang, \& Seedig, 2015).

Moulin (1986) discussed the voting tree rule which is a generalization of the amendment procedure. This general rule employs a binary voting tree where

- the leaves represent the alternatives such that each alternative is represented by at least one leaf, and

- each internal node represents the alternative that wins the pairwise comparison of its children.

The alternative represented by the root defines the winner. If the binary tree is degenerate (that is, at least one child of each internal node is a leaf) and if each alternative is represented by exactly one leaf, then this procedure is identical to the amendment procedure. To tackle Coalitional Manipulation with weighted voters, Conitzer et al. (2007) provided a cubic-time algorithm for the voting tree procedure while our quadratic-time algorithm (Theorem 4) is tailored for the amendment procedure. Xia and Conitzer (2011) provided intractability results for the Possible (resp. NeCEssary) Winner problem with weighted voters when the given tree is balanced. Pini, Rossi, Venable, and Walsh (2011) and Lang, Pini, Rossi, Salvagnin, Venable, and Walsh (2012) showed that Possible (resp. Necessary) Winner with weighted voters is NP-complete (resp. coNP-complete) even for a constant number of voters (see Table 1 for an overview of known and our new results).

\subsection{Our Contributions}

We investigate computational problems for two prominent parliamentary voting procedures: the successive procedure and the amendment procedure. We study three types of voting problems. First, we study the AGEnda Control problem that asks whether there is an agenda under which a given alternative can win when the voters vote according to their preferences. Second, we study the Coalitional Manipulation problem that asks whether a given alternative can win by adding a given number of voters. Third, we study whether 


\begin{tabular}{llll}
\hline Problem & Successive & Amendment & References \\
\hline Agenda Control & $O\left(n \cdot m^{2}\right)$ & $O\left(n \cdot m^{2}+m^{3}\right)^{\ominus}$ & Thm. $1 \& 2$ \\
W. Coalitional Manipulation & $O(n \cdot m)$ & $O\left(n \cdot m^{2}\right)$ & Thm. $3 \& 4$, Cor. 1 \\
Possible Winner & NP-c & NP-c & Thm. $5 \& 6$ \\
& ILP-FPT $(m)$ & ILP-FPT $(m)$ & Cor. 2 \\
NeCESSARY Winner & $O\left(n \cdot m^{3}\right)$ & coNP-c & Thm. $8 \& 9$ \\
& & ILP-FPT $(m)$ & Cor. 3 \\
W. Possible Winner & NP-c $(m=3)$ & NP-c $(m=3)$ & Thm. 10 \\
W. Necessary Winner & $O\left(w \cdot n \cdot m^{3}\right)$ & $O(n)$ for $m \leq 3$ & Thm. 11 \\
& & coNP-c $(m=4)$ & - \\
\hline
\end{tabular}

Table 1: Computational complexity results of this paper and of the literature for our two parliamentary voting procedures. The prefix "W." indicates that the relevant problem has weighted voters. The number of voters is denoted by $n$, the number of alternatives is denoted by $m$, the number of manipulators is denoted by $k$, and the sum of weights (encoded in binary) of all voters in an instance of the COALITIONAL MANIPULATION problem is denoted by $w$. "ILP-FPT $(m)$ " stands for "fixed-parameter tractable with respect to $m$ due to a formulation as an integer linear program". In particular, this means that if the number $m$ of alternatives is a constant, then the respective problem is polynomial-time solvable and the degree of the polynomial does not depend on $m$. The result marked with ${ }^{\circ}$ also follows from the work of Miller (1977). Results marked with follow from the work of Pini et al. (2011) and Lang et al. (2012). Entries containing statements of the form "NP-c $(m=z)$ " (resp. "coNP-c $(m=z)$ ") mean that the relevant problem is in NP (resp. coNP) and is NP-hard (resp. coNP-hard) even with only $z$ many alternatives. All hardness results hold even when the agenda is a linear order. The NP-hardness result for POssible Winner with weighted voters is implied by the weakly NP-hardness result, stated in Theorem 10. All NP (resp. coNP) membership results follow from the fact that we can non-deterministically guess the respective completion of the profile and of the agenda and deterministically compute the winner in polynomial time.

a given alternative can possibly (resp. necessarily) win when the voters may have incomplete preferences; we call the corresponding problem Possible Winner (resp. Necessary Winner). See Table 1 for an overview of our theoretical results.

Our polynomial-time algorithms for Agenda Control and Coalitional ManipuLATION indicate that in the worst-case, the amendment procedure is computationally more expensive than the successive procedure. From a computational perspective, this implies that the amendment procedure may be more resistant to manipulation and agenda control than the successive procedure.

We also study Agenda Control and Coalitional Manipulation empirically. Our experiments on Agenda Control and on Coalitional Manipulation using real-world profiles indicate that while both problems are polynomial-time solvable, a successful agenda 
control is very rare and a successful manipulation on average needs a coalition containing at least half of the original voters. Thus, we may conclude that both parliamentary voting procedures are still "safe" against these voting attacks in the real world.

If voters' preference orders are incomplete, then deciding whether an alternative possibly wins is NP-complete for both procedures while deciding whether an alternative necessarily wins is polynomial-time solvable for the successive procedure, but is coNP-complete for the amendment procedure. We also show that all problems with hardness results can be solved in polynomial time when the number $m$ of alternatives is a constant, and the degree of the corresponding polynomial does not depend on $m$ (in other words, this means fixedparameter tractability with respect to the parameter $m$ ).

\subsection{Organization of the Paper}

In Section 2, we provide definitions regarding voter preferences, preference profiles, and our central parliamentary voting procedures.

In Section 3, we focus on AgEnda Control when voters have sincere preference orders and we study the corresponding computational complexity for both parliamentary voting procedures. In Section 4, we deal with strategic behavior of voters and investigate the computational complexity of Coalitional Manipulation. In Section 5, we complement our theoretical study with an experimental evaluation of Agenda Control and CoAlitional MANIPULATION for real-world profiles.

In Section 6, we consider situations with uncertainty, that is, when voters' preferences and the agenda are still incomplete. We study the problem of determining whether an alternative can possibly/necessarily win in such a situation (the POSSIBLE WINNER problem/the Necessary Winner problem). Section 7 concludes our work and presents some challenges for future research.

\section{Basic Notations, Definitions, and Examples}

First of all, for general expositions on computational social choice, we refer to the books of Rothe (2015) and Brandt, Conitzer, Endriss, Lang, and Procaccia (2016).

Let $A:=\left\{a_{1}, \ldots, a_{m}\right\}$ be a set of $m$ alternatives. A preference order $\succ$ over the alternatives $A$ is a partial order over $A$ (a reflexive, anti-symmetric, and transitive binary relation). For two distinct alternatives $b, c \in A$, the relation $b \succ c$ means that $b$ is strictly preferred to $c$. If neither $(b, c) \in \succ$ nor $(c, b) \in \succ$ holds, then we say that $b$ and $c$ are incomparable to each other and write $b \|_{\succ c}$.

Let $V$ be a set of $n$ voters, where each voter $v_{i} \in V$ has a preference order $\succ_{i}$ over $A$. A preference profile $\mathcal{P}:=\left(A, V,\left(\succ_{1}, \succ_{2}, \cdots, \succ_{n}\right)\right)$ is a tuple consisting of the set $A$ of alternatives, the set $V$ of voters, and the collection $\left(\succ_{1}, \succ_{2}, \ldots, \succ_{n}\right)$ of preference orders for the voters in $V$. Given two disjoint subsets $B, C \subseteq A$ of alternatives, we write $B \succ_{i} C$ to express that voter $v_{i}$ prefers set $B$ to set $C$, that is, for each alternative $b \in B$ and each

alternative $c \in C$ it holds that $b \succ_{i} c$. If not stated explicitly, then we write $\vec{B}$ to denote an arbitrary but fixed preference order over $B$. We write $\overleftarrow{B}$ to denote the corresponding reverse order. 
We say that alternative $b$ beats alternative $c$ (in a head-to-head contest) when a majority of voters prefers $b$ to $c$. We call $b$ a Condorcet winner if it beats every other alternative. By definition, Condorcet winners are unique but do not always exist when the number of voters is even.

Let $\mathcal{P}^{\prime}:=\left(A^{\prime}, V^{\prime},\left(\succ_{1}^{\prime}, \succ_{2}^{\prime}, \cdots, \succ_{n}^{\prime}\right)\right)$ be a second profile for the same set of alternatives and the same set of voters. If for each voter $v_{i} \in V$, each preference order $\succ_{i}^{\prime}$ from $\mathcal{P}^{\prime}$ is a superset of the preference order $\succ_{i}$ from $\mathcal{P}$, then we say that $\mathcal{P}^{\prime}$ extends $\mathcal{P}$. Note that a preference order is in effect a set of ordered pairs of alternatives. If each $\succ_{i}^{\prime}$ is even a linear order, then we say that $\mathcal{P}^{\prime}$ completes $\mathcal{P}$.

We can use a directed graph to illustrate the comparisons between every two alternatives. We provide an example for this concept in Example 1.

Definition 1 ((Weighted) majority graph). Given a preference profile $\mathcal{P}:=\left(A, V, \succ_{1}, \succ_{2}\right.$ $\left.\left., \cdots, \succ_{n}\right)\right)$, we construct an arc-weighted directed graph $G=(U, E)$, where $U$ consists of a vertex $u_{j}$ for each alternative $a_{j} \in A$ and where there is an arc from vertex $u_{j}$ to vertex $u_{j^{\prime}}$ with weight $w$ if exactly $w$ voters prefer $a_{j}$ to $a_{j^{\prime}}$. We call the constructed graph $G$ a weighted majority graph for profile $\mathcal{P}$.

If we ignore the weights and the arcs with weights of at most $|V| / 2$, then we obtain a majority graph (without weights) for the profile $\mathcal{P}$. We call the constructed majority graph a tournament if there is exactly one arc for every two distinct vertices.

Next, we give a small example to illustrate the concept of (weighted) majority graphs.

Example 1. Let $\mathcal{P}$ be a preference profile with three alternatives $a, b, c$, and three voters $v_{1}, v_{2}, v_{3}$ whose preference orders are specified as follows:

$$
v_{1}: a \succ b \succ c, \quad v_{2}: b \succ a \succ c, \quad v_{3}: c \succ a \succ b .
$$

The weighted majority graph for $\mathcal{P}$ consists of three alternatives and six weighted arcs as depicted in the left figure below. Bold arcs indicate the majority relation that a majority of voters prefers the source of the arc to the target of the arc.
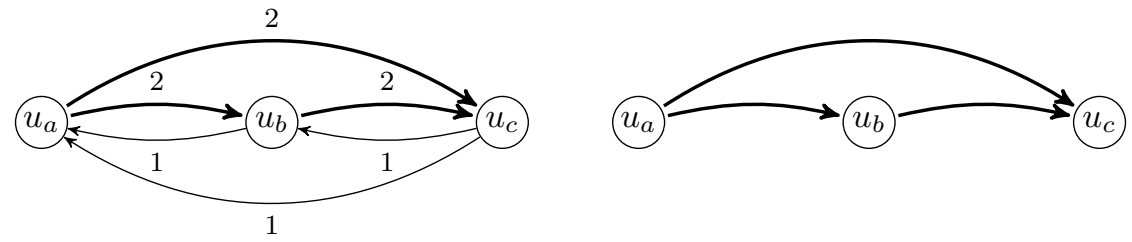

The corresponding majority graph without weights is depicted in the right figure. The graph shown in the right figure is a tournament. Further, vertex $u_{a}$ has two out-going arcs, meaning that alternative $a$ beats the other two alternatives. Thus, it is a Condorcet winner.

\subsection{The Successive and the Amendment Procedures}

We consider two of the most common parliamentary voting procedures. For both procedures, we assume that a linear order over the $m$ alternatives in $A$ is given. We refer to this linear order $\mathcal{L}$ as an agenda. If this order is a partial order, then we call it a partial agenda, denoted by $\mathcal{B}$. We use the symbol $\triangleright$ for the agenda order to distinguish it from the 
preference order $\succ$ : For two distinct alternatives $a$ and $b, a \triangleright b$ means that $b$ is considered after (or ordered behind) $a$ in the agenda.

Roughly speaking, the successive procedure determines a winner that is the first alternative in the agenda $\mathcal{L}$ such that a majority of voters prefers it to every alternative ordered behind it in $\mathcal{L}$.

Definition 2 (Successive procedure for a given agenda $\mathcal{L}$ ). There are at most $m$ rounds with $m$ being the number of alternatives. Starting with round $i:=1$, we repeat the following until we find a successive winner: Let $c$ be the $i^{\text {th }}$ alternative in $\mathcal{L}$. If there is a majority of voters who prefer alternative $c$ to every alternative that is ordered behind it in $\mathcal{L}$, then $c$ is the successive winner. Otherwise, we proceed to round $i:=i+1$.

In Europe, the successive procedure is used in many parliamentary chambers including those of Austria, Belgium, Denmark, France, Germany, Greece, Iceland, Ireland, Italy, Luxembourg, the Netherlands, Norway, Portugal, and Spain (Rasch, 2000). We give a small example to illustrate how the successive procedure works.

Example 2. Let us look at the profile $\mathcal{P}$ from Example 1 again. Consider the following agenda $\mathcal{L}: a \triangleright b \triangleright c$ for the successive procedure. We have three alternatives. Thus, the procedure ends after at most three rounds. In the first round, since less than half of the voters prefer $a$ to both $b$ and $c$ (only $v_{1}$ does), $a$ is not a successive winner, although it is a Condorcet winner. In the second round, since more than half of the voters prefer $b$ to $c$ (voters $v_{1}$ and $v_{2}$ ), $b$ is the successive winner.

The amendment procedure determines a winner that is the first alternative in the agenda $\mathcal{L}$ to beat every other alternative considered after it in $\mathcal{L}$. In other words, an amendment winner is the first alternative that becomes a Condorcet winner if all alternatives considered before it in the agenda are eliminated from the profile.

Definition 3 (Amendment procedure for a given agenda $\mathcal{L}$ ). It proceeds in $m$ rounds with $m$ being the number of alternatives. We define an amendment winner for each round. Let the $1^{\text {st }}$-round amendment winner be the $1^{\text {st }}$ alternative in $\mathcal{L}$. Then, for each round $i, 2 \leq i \leq m$, the $i^{\text {th }}$-round amendment winner is either the $(i-1)^{\text {th }}$-round amendment winner $b$ or the $i^{\text {th }}$ alternative $c$ in $\mathcal{L}$ : if a majority of voters prefers $b$ over $c$, then it is $b$. Otherwise it is $c$. We define the $m^{\text {th }}$-round amendment winner to be the amendment winner.

In Europe, the amendment procedure is used in the parliamentary chambers of Finland, Sweden, Switzerland, and the United Kingdom. It is also used in the U.S. Congress and several other countries with Anglo-American ties (Rasch, 2000). We again use Example 1 to illustrate how the amendment rule works. It may be helpful to consider the corresponding majority graph (see Definition 1).

Example 3. Consider the profile given in Example 1 and the agenda $\mathcal{L}: a \triangleright b \triangleright c$ given in Example 2. Alternative $a$ is the $1^{\text {st }}$-round winner since it is the first alternative in $\mathcal{L}$. Since a majority of voters prefers $a$ to $b$ and a majority of voters prefers $a$ to $c$, alternative $a$ is both the $2^{\text {nd }}$-round and the $3^{\text {rd }}$-round winner. Hence, it is the amendment winner. Indeed, as shown in the majority graph (see Example 1), alternative $a$ is a Condorcet winner.

As already observed by Miller (1977), Condorcet winners are always amendment winners: 
Observation 1 (Miller, 1977). A Condorcet winner is an amendment winner, no matter what the agenda looks like.

We close this section with some remarks. For the remainder of this paper, we assume that the number of voters is odd to reduce the impact of tie-breaking and we leave the study of different tie-breaking rules as future work. We consider both unweighted voters and voters with integer weights. The weighted case is especially interesting in the parliamentary setting: First, there are parliamentary chambers where voters are weighted (for instance, in the Council of Europe, preference orders are weighted according to the population size of the country). Second, voters will often vote along party lines. This effectively gives us parties casting weighted preference orders. Third, the weighted case can inform the situation where we have uncertainty about the preference orders. For example, Conitzer et al. (2007, Thm. 15) proved that if the Coalitional Manipulation problem for a voting rule is NPhard for weighted voters with complete preference orders, then deciding who possibly wins in the unweighted case is NP-hard even when there is only a limited form of uncertainty about the preference orders. It would be interesting to prove similar results about uncertainty and weighted preference orders for parliamentary voting procedures.

\section{Agenda Control}

The order of the alternatives, that is, the agenda, may depend on the speaker, the Government, logical considerations (for instance, the status quo goes last, the most extreme alternative comes first), the chronological order of submission, or other factors. The agenda used can have a major impact on the final decision. It is also worth noting that there are many possible agendas.

For example, if we use the amendment procedure, then the Condorcet winner is the only amendment winner (Observation 1). But the Condorcet winner is only guaranteed to win the successive procedure if it is introduced in one of the last two positions in the agenda. To illustrate this fact, consider the profile given in Example 1 again. If alternative $a$ is the first to be considered in the agenda, then $a$ will be eliminated because it is not a majority winner (with respect to the rest). Thus, it cannot become a successive winner. However, if $a$ is not the first one to consider in the agenda, then it will become a successive winner because the first one (either $b$ or $c$ ) in the agenda will be eliminated and in the next round where two alternatives (including $a$ ) remain, $a$ will beat the other remaining one.

We therefore study how hard is it for an agenda setter to control the outcome of a parliamentary procedure when the preferences of the voters are linear and known. In particular, we consider the following computational question.

\section{Agenda Control}

Input: A preference profile $\mathcal{P}:=\left(A, V,\left(\succ_{1}, \succ_{2}, \cdots, \succ_{n}\right)\right)$ with linear preference orders $\succ_{i}$ and a preferred alternative $p \in A$.

Question: Is there an agenda for $A$ such that $p$ is the overall winner?

We find that both voting procedures are "vulnerable" to agenda control. In particular, we show how to find in polynomial time an appropriate agenda (if it exists) such that the preferred alternative can become a successive (resp. amendment) winner. In the remainder 
of this section, we assume that a preference profile $\mathcal{P}:=\left(A, V,\left(\succ_{1}, \succ_{2}, \cdots, \succ_{n}\right)\right)$ with linear preference orders and a preferred alternative $p \in A$ are given. We assume that the voters vote sincerely according to their linear preference orders. If the agenda is not revealed in advance, risk averse voters are likely to vote sincerely. Note that this computational question also applies to the situation where voters are strategic, the chair collects there possibly insincere votes, and then decides on an agenda to give a particular outcome. Recall that $n$ denotes the number of voters and $m$ denotes the number of alternatives in a given profile $\mathcal{P}$.

\subsection{The Successive Procedure}

The basic approach to controlling the successive procedure is to build an agenda in reverse order such that each of the alternatives that are currently among the highest positions in the partial agenda may be strong enough to beat $p$ alone but is too weak to be a majority winner against the whole set of alternatives behind it. To formalize this idea, we need the notion of majority winner with respect to a subset of alternatives.

Definition 4 ( $A^{\prime}$-majority winners). Let $A^{\prime} \subseteq A$ be a subset of alternatives. An alternative $a \in A^{\prime}$ from the set $A^{\prime}$ is an $A^{\prime}$-majority winner if there is a majority of voters who each prefer $a$ to every alternative from $A^{\prime} \backslash\{a\}$.

Note that a Condorcet winner for a subset $A^{\prime} \subseteq A$ of alternatives is not necessarily an $A^{\prime}$-majority winner as Example 1 shows: Alternative $a$ is a Condorcet winner but not an $\{a, b, c\}$-majority winner.

Next, we derive necessary conditions for an alternative to be a successive winner.

Observation 2. Let $\mathcal{L}$ be an agenda for the alternatives $A$, let $A^{\prime} \subset A$ be a proper subset of alternatives, and let $b \in A \backslash A^{\prime}$ be an arbitrary alternative. If $b$ is considered before all alternatives in $A^{\prime}$ by $\mathcal{L}$ and if $b$ is not an $\left(A^{\prime} \cup\{b\}\right)$-majority winner, then $b$ cannot be $a$ successive winner.

Proof. By the definition of the successive procedure, if $b$ would be a successive winner for agenda $\mathcal{L}$, then it would have been an $\left(A^{\prime} \cup\{b\}\right)$-majority winner since it is considered before all alternatives in $A^{\prime}$ by the agenda - a contradiction.

We can generalize Observation 2 to hold for a subset of alternatives.

Lemma 1. Let $A^{\prime} \subset A$ be a proper subset of alternatives. If every alternative $b$ in $A \backslash A^{\prime}$ is an $\left(A^{\prime} \cup\{b\}\right)$-majority winner, then no alternative from $A^{\prime}$ can be a successive winner.

Proof. Suppose for the sake of contradiction that there is an agenda $\mathcal{L}$ for which the successive winner $a^{\prime}$ would come from $A^{\prime}$. By the definition of the successive procedure, this would imply that a majority of voters prefers $a^{\prime}$ over every alternative that is considered behind $a^{\prime}$ in $\mathcal{L}$. However, by the assumption that every alternative $b$ in $A \backslash A^{\prime}$ is an $\left(A^{\prime} \cup\{b\}\right)$-majority winner and that $a^{\prime} \in A^{\prime}$, it must hold that all alternatives in $A \backslash A^{\prime}$ are considered before $a^{\prime}$ in $\mathcal{L}$, that is, $\mathcal{L}:\left(A \backslash A^{\prime}\right) \triangleright a^{\prime}$. Let $d$ be the last alternative in $A \backslash A^{\prime}$ that is still in front of $a^{\prime}$, that is,

$$
\mathcal{L} \text { satisfies }\left(\left(A \backslash A^{\prime}\right) \backslash\{d\}\right) \triangleright d \triangleright a^{\prime} .
$$




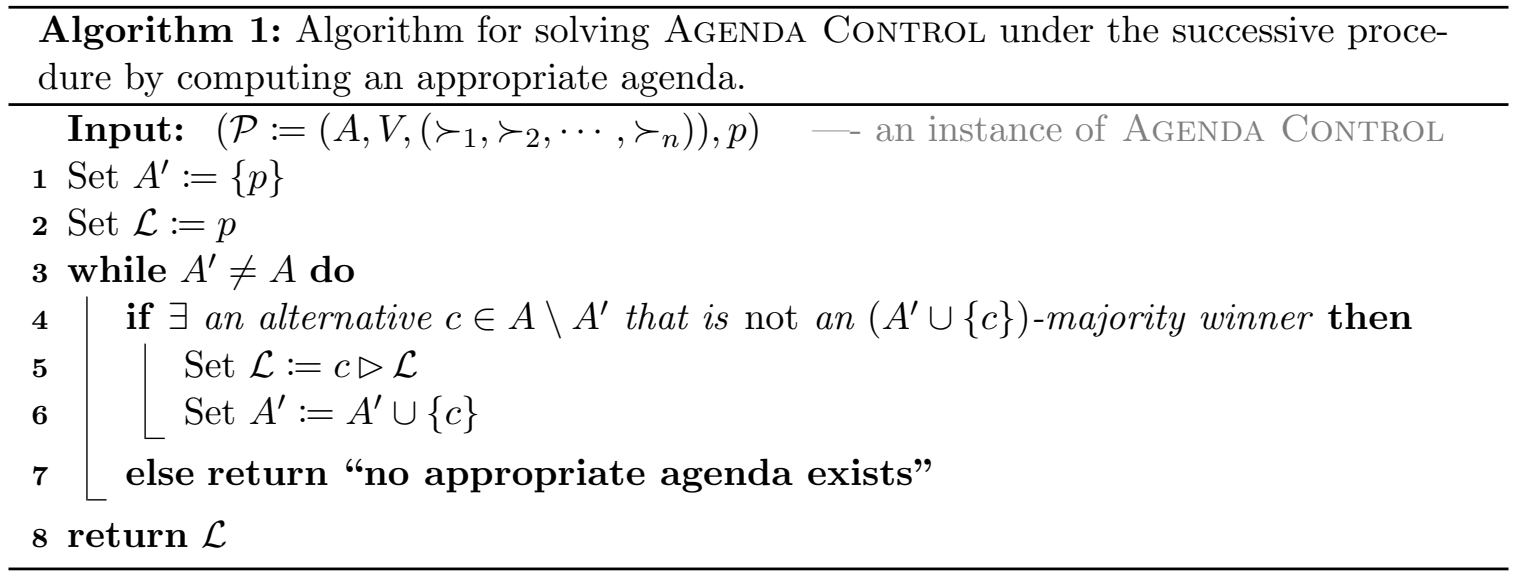

But, since $a^{\prime}$ is a successive winner, there must be a round when $d$ is considered and $d$ would become a successive winner (note that all alternatives behind $d$ come from $A^{\prime}$ ) - a contradiction.

By Lemma 1, we can construct an agenda in reverse order by first placing our preferred alternative $p$ in the last position and setting $A^{\prime}:=\{p\}$. We will extend the agenda by putting all alternatives $c$ that are not $\left(A^{\prime} \cup\{c\}\right)$-majority winners right in front of $A^{\prime}$. Then, we update the set $A^{\prime}:=A^{\prime} \cup\{c\}$. Using this approach we can solve the AGEndA Control problem for the successive procedure in polynomial time.

Theorem 1. For the successive procedure, solving AGENDA CONTROL and finding an agenda for a yes-instance of AGENDA CONTROL can be done in $O\left(n \cdot m^{2}\right)$ time, where $n$ denotes the number of voters and $m$ denotes the number of alternatives.

Proof. Given a profile $\mathcal{P}$ and an alternative $p$, we use Algorithm 1 to either decide that $p$ cannot be a successive winner or construct an agenda for which $p$ wins.

For the correctness of the algorithm, if the condition in Line 4 does not apply, then by Lemma 1 , no alternative in $A^{\prime}$ can win, implying that $p$ can never win because $p \in A^{\prime}$. Thus, we can safely reply with "no".

Now, suppose that the condition in Line 4 always applies. We show that if $p$ is a successive winner of a profile restricted to the alternative set $A^{\prime}$, then it is also a successive winner of the restricted profile that additionally contains an alternative $c$ that is a non$\left(A^{\prime} \cup\{c\}\right)$-majority winner. To this end, let $c$ be an alternative in $A \backslash A^{\prime}$ that is not an $\left(A^{\prime} \cup\{c\}\right.$ )-majority winner (see Definition 4). Assume that $p$ is a successive winner under the current agenda $\mathcal{L}$ for the profile $\mathcal{P}^{\prime}$ restricted to the alternatives in $A^{\prime}$. Then, by Observation 2, it follows that under every agenda that extends $c \triangleright A^{\prime}, c$ is not a successive winner. This means that the procedure would delete $c$ and go on with the alternatives in $A^{\prime}$. By assumption, $p$ is a successive winner for profile $\mathcal{P}^{\prime}$ and agenda $\mathcal{L}$. Therefore, in the profile restricted to the alternatives in $A^{\prime} \cup\{c\}$, the agenda $c \triangleright \mathcal{L}$ also makes $p$ win. This completes the correctness proof.

Finally, we come to the running time analysis. First, the procedure inside the while loop (Lines 3 and 7 ) is executed at most $m$ times. Second, inside the while loop, for every 
alternative $c \in A \backslash A^{\prime}$, we check whether it is an $A^{\prime} \cup\{c\}$-majority winner. This check can be done in $O(n)$ time: We maintain a list $T$ of size $n$ that, for each voter $v$, stores the highest position of alternative from $A^{\prime}$ ranked by $v$. We iterate over each voter $v$ and compare the position $v(c)$ of $c$ ranked by $v$ and the position $T(v)$ stored by $T$ for voter $v$. We count the number of times where $v(c)<T(v)$. If this number is smaller than $n / 2$, then $c$ is not an $A^{\prime} \cup\{c\}$-majority winner; we add $c$ to $A^{\prime}$ and we update the list $T$ by changing the entry $T(v)$ to $v(c)$ if $v(c)<T(v)$. Altogether, the running time is $O\left(n \cdot m^{2}\right)$.

\subsection{The Amendment Procedure}

Controlling the amendment procedure is closely related to finding a Hamiltonian cycle in a strongly connected tournament ("strongly connected" means that for each two vertices $u$ and $v$, there is a directed path from $u$ to $v$ ). To see this, we first construct a majority graph for the given preference profile (see the corresponding definition in Section 2). Recall that

we assume the number of voters to be odd. The majority graph has $m$ vertices and $\left(\begin{array}{c}m \\ 2\end{array}\right)$ arcs and is indeed a tournament. From the theory of directed graphs (Harary \& Moser, 1966, Thm. 7), we know that every strongly connected tournament contains a Hamiltonian cycle.

Observation 3 (Harary \& Moser, 1966, Thm.7). Every strongly connected tournament contains a Hamiltonian cycle.

By carefully examining the constructive proof for Observation 3, we can find a Hamiltonian cycle in $O\left(m+m^{2}+m \cdot\left(m+m^{2}\right)\right)=O\left(m^{3}\right)$ time.

Now, the crucial idea is to check whether the vertex that corresponds to $p$ belongs to a strongly connected component that has only out-going arcs. Alternative $p$ can win under an appropriate agenda if and only if this is the case.

Using depth-first search (see Cormen et al., 2009), every directed graph can be partitioned into strongly connected components in linear time. Since our majority graph is not only directed but also a tournament, we can easily obtain the following result.

Observation 4. Every tournament can be partitioned into disjoint strongly connected components such that the following holds.

1. Each component is a subtournament.

2. All arcs from the vertices of a component to another have only one direction.

3. The graph resulting from deleting all but one arbitrary vertex from each component is acyclic and a tournament.

From this observation, we can derive the next theorem. Note that Miller (1977) already characterized the set of alternatives that can become an amendment winner under an appropriate agenda. Our theorem strengthens this result by giving a polynomial-time algorithm to construct the desired agenda.

Theorem 2. For the amendment procedure, solving Agenda Control and finding an agenda for a yes-instance of AGENDA CONTROL can be done in $O\left(n \cdot m^{2}+m^{3}\right)$ time, where $n$ denotes the number of voters and $m$ denotes the number of alternatives. 
Proof. By Observation 4, every tournament consists of strongly connected subtournaments which can be ordered by topological sorting. Now, observe that only the alternatives corresponding to the vertices from the top-most strongly connected subtournament ${ }^{1}$ can become an amendment winner. In other words, if the vertex corresponding to the preferred alternative $p$ does not belong to the top-most subtournament, then $p$ can never win. As already mentioned, Observation 3 is constructive and we can find a Hamiltonian cycle for a strongly connected tournament with $m$ vertices in $O\left(\mathrm{~m}^{3}\right)$ time. Now, we construct a sequence $L_{\text {ver }}$ of vertices by reversing the orientation of the Hamiltonian cycle, starting with the predecessor of the vertex $u_{p}$ corresponding to $p$ and ending at the vertex $u_{p}$, and let $L_{\text {alt }}$ be the order of the alternatives corresponding to $L_{\mathrm{ver}}$. We can verify that $p$ is an amendment winner for every agenda that extends order $L_{\text {alt }}$.

Thus, the problem is reduced to finding strongly connected subtournaments of the majority graph for the given preference profile: If the vertex corresponding to $p$ is in the top-most subtournament, then construct an arbitrary but fixed order that extends $L_{\text {alt }}$ and we answer "yes" by returning $L_{\text {alt }}$. Otherwise we answer "no".

Now, we come to the running time. Constructing a majority graph for a profile takes $O\left(n \cdot m^{2}\right)$ time; note that the majority graph will have $O(m)$ vertices and $O\left(m^{2}\right)$ arcs. Partitioning the majority graph into strongly connected components takes $O\left(\mathrm{~m}^{2}\right)$ time and checking whether the vertex corresponding to $p$ belongs to the top-most component takes $O(m)$ time. Finally, finding a Hamiltonian cycle in a strongly connected tournament takes $O\left(\mathrm{~m}^{3}\right)$ time. Thus, in total, our algorithm for solving Agenda Control for the amendment procedure takes $O\left(n \cdot m^{2}+m^{3}\right)$ time.

We close this section with two remarks. First, the algorithm for the successive procedure actually works for both odd and even numbers of voters; for even number $n$ voters, a majority of voters simply means more than $n / 2$ voters. Second, our algorithm for the amendment procedure can be extended to the case where the number of voters is even. There, alternative $p$ is a winner if and only if no strongly connected component "dominates" (that is, is preferred by a majority of voters) the strongly connected component that contains vertex $u_{p}$; if two alternatives are tied in a head-to-head contest, then we break ties in favor of the alternative that is considered later.

\section{Manipulation}

In this section, we consider the question of how difficult it is for a coalition of voters to vote strategically to ensure a given outcome, assuming that the other voters vote sincerely. Specifically, we focus on the case where the desired outcome is to make a given alternative win. We provided polynomial-time algorithms for both our voting procedures (Theorems 3 and 4$)$.

In social choice theory, it is also interesting to study a weaker goal, which is to make any alternative win that is better (compared to the current winner) for the manipulators. It is straightforward to see that our polynomial-time algorithms, showing Theorems 3 and 4, can be adapted to check whether the weaker goal can be achieved.

1. The vertices of the top-most strongly connected subtournament are also known as Good set (1971), Schwartz set (1972), or Smith set (1973). 
Coalitional Manipulation

Input: A profile $\mathcal{P}:=\left(A, V,\left(\succ_{1}, \succ_{2}, \cdots, \succ_{n}\right)\right)$ with linear preference orders $\succ_{i}$, a preferred alternative $p \in A$, a non-negative integer $k \in \mathbb{N}$, and an agenda $\mathcal{L}$ for $A$.

Question: Is it possible to add a set of $k$ voters (a coalition) such that $p$ wins under agenda $\mathcal{L}$ ?

We find that deciding whether a manipulation is successful is polynomial-time solvable for both the successive procedure and the amendment procedure. However, our approach to deciding whether the amendment procedure can be successfully manipulated has a running time that is asymptotically higher than our approach to deciding the same question for the successive procedure.

First, we observe that the manipulators can basically vote in the same way.

Observation 5. For the successive procedure as well as the amendment procedure, if there is a successful (weighted) manipulation, then there is also a successful one where all voters from the coalition rank the alternatives in the same way.

Proof. For the successive procedure, if there is a successful manipulation for the preferred alternative $p$, then requiring all manipulators to rank $p$ in the first position and to rank the other alternatives in an arbitrary but fixed order also makes $p$ win.

Now, let $\mathcal{P}^{\prime}$ be a manipulated profile, that is, the original profile plus the manipulators. Let $X:=\left\{x_{1}, x_{2}, \ldots, x_{s}\right\}$ consist of all alternatives $x_{i} \in A$ such that there is a round index $r_{i}$ where $x_{i}$ is an $r_{i}^{\text {th }}$-round amendment winner in profile $\mathcal{P}^{\prime}$. Assume without loss of generality that for every $2 \leq i \leq s$, alternative $x_{i}$ beats $x_{i-1}$. If $p$ is an amendment winner in profile $\mathcal{P}^{\prime}$, then $p=x_{s}$. Furthermore, we can verify that each $x_{i}$ is an $r_{i}^{\text {th }}$-round amendment winner in the original profile plus the $k$ manipulators who all have preference order $x_{s} \succ x_{s-1} \succ \ldots \succ x_{1} \succ \overrightarrow{A \backslash X}$. This implies that $x_{s}=p$ is an amendment winner.

As in the proof for Observation 5, an optimal way of manipulating the successive procedure is to let the manipulators rank their most preferred alternative $p$ at the first position. Using this fact, we obtain a simple linear-time algorithm for manipulating the successive procedure.

Theorem 3. For the successive procedure, solving COALitional Manipulation and, for yes-instances additionally constructing the preference order for the manipulators can be done in $O(n \cdot m)$ time, where $n$ denotes the number of voters and $m$ denotes the number of alternatives.

Proof. In the proof of Observation 5, we can observe that if a coalition of $k$ voters can manipulate the successive procedure, then ranking alternative $p$ in the first position and the other alternatives in an arbitrary but fixed order can also make $p$ win. This leads to a linear-time $O((k+n) \cdot m)$ algorithm: Let the coalition all vote $p \succ \overrightarrow{A \backslash\{p\}}$, and check whether $p$ wins under the successive procedure. Since a coalition that is larger than the set of original voters can always make $p$ win, we can assume that $k<n+1$ which leads to $O((n \cdot m)$ time.

For the successive procedure, we have seen that how the manipulators should vote does not depend on the sequence of the alternatives in the agenda. For the amendment 
procedure, however, a successful manipulation greatly depends on the agenda. To elaborate this observation, we need the following.

Definition $5\left(i^{\text {th }}\right.$-round manipulation winners $)$. Let $\left(\mathcal{P}:=\left(A, V,\left(\succ_{1}, \succ_{2}, \cdots, \succ_{n}\right)\right), p, k, \mathcal{L}\right)$ be an instance of CoAlitional Manipulation under the amendment procedure. We call an alternative an $i^{\text {th }}$-round manipulation winner if adding some coalition of $k$ additional voters to the original profile $\mathcal{P}$ makes this alternative the $i^{\text {th }}$-round amendment winner under agenda $\mathcal{L}$.

We give an example to illustrate the notion of an $i^{\text {th }}$-round manipulation winner.

Example 4. Consider the following profile with four alternatives $a, b, c, d$ and three voters $v_{1}, v_{2}, v_{3}$. The preference orders of these three voters are given as follows:

$$
v_{1}: a \succ b \succ c \succ d, \quad v_{2}: b \succ c \succ a \succ d, \quad v_{3}: d \succ b \succ c \succ a .
$$

Let the agenda be $\mathcal{L}: a \triangleright b \triangleright c \triangleright d$. Suppose that there are two manipulators. First of all, alternative $a$ is the only $1^{\text {st }}$-round manipulation winner. Both alternatives $a$ and $b$ are the $2^{\text {nd }}$-round manipulation winners: The second alternative in $\mathcal{L}$ is $b$. If the two added manipulators have the same preference order $a \succ b \succ c \succ d$, then $a$ beats $b$. If they have the preference order $b \succ a \succ c \succ d$, then $b$ beats $a$.

By the definition of $i^{\text {th }}$-round manipulation winners, we know that an alternative that is not an $i^{\text {th }}$-round manipulation winner can never become a $j^{\text {th }}$-round manipulation winner with $j>i$. Further, one can efficiently check whether an $i^{\text {th }}$-round manipulation winner alternative can survive as a manipulation winner of a later round, as the following shows.

Lemma 2. Let $b$ be the $i^{\text {th }}$ alternative in agenda $\mathcal{L}, 2 \leq i \leq m$.

1. Alternative $b$ is an $i^{\text {th }}$-round manipulation winner if and only if there is an $(i-1)^{\text {th }}$ round manipulation winner $c$ such that requiring all manipulators to prefer $b$ to $c$ makes b beat $c$.

2. An $(i-1)^{\text {th }}$-round manipulation winner $c$ is also an $i^{\text {th }}$-round manipulation winner if and only if requiring all manipulators to prefer $c$ to $b$ makes $c$ beat $b$.

Proof. We only show the first statement as the second one can be shown analogously. Let $A$ be the set of all alternatives. For the "only if" part, assume that $b$ is an $i^{\text {th }}$-round manipulation winner and let $\mathcal{P}^{\prime}$ be the profile with the additional manipulators for which $b$ is an $i^{\text {th }}$-round amendment winner. We rename and enumerate all $j^{\text {th }}$-round amendment winners in $\mathcal{P}^{\prime}$ with $j \leq i-1$ as $x_{1}, x_{2}, \ldots, x_{s}$ so that for each $1 \leq \ell \leq s-1$, alternative $x_{\ell+1}$ beats $x_{\ell}$ in $\mathcal{P}^{\prime}$. By definition, $x_{s}$ is the $(i-1)^{\text {th }}$-round amendment winner. Let $X:=$ $\left\{x_{1}, x_{2}, \ldots, x_{s}\right\}$. We can verify that $b$ is an $i^{\text {th }}$-round amendment winner in the manipulated profile where every manipulator has preference order $b \succ x_{s} \succ x_{s-1} \succ \ldots \succ x_{1} \succ \overrightarrow{A \backslash X}$ with $A \backslash X$ being the remaining alternatives.

For the "if" part, let $c$ be an $(i-1)^{\text {th }}$-round manipulation winner and consider the manipulators' preference orders $\succ_{1}^{\prime}, \succ_{2}^{\prime}, \ldots, \succ_{k}^{\prime}$ that only rank the first $(i-1)$ alternatives in the agenda. If requiring every manipulator to prefer $b$ to $c$ makes $b$ beat $c$, then at round $i$, alternative $b$ will survive as the $i^{\text {th }}$-round amendment winner when every manipulator $i$ has a preference order extending $\succ_{i}^{\prime} \cup\{b \succ c\}$. This implies that $b$ is an $i^{\text {th }}$-round manipulation winner. 


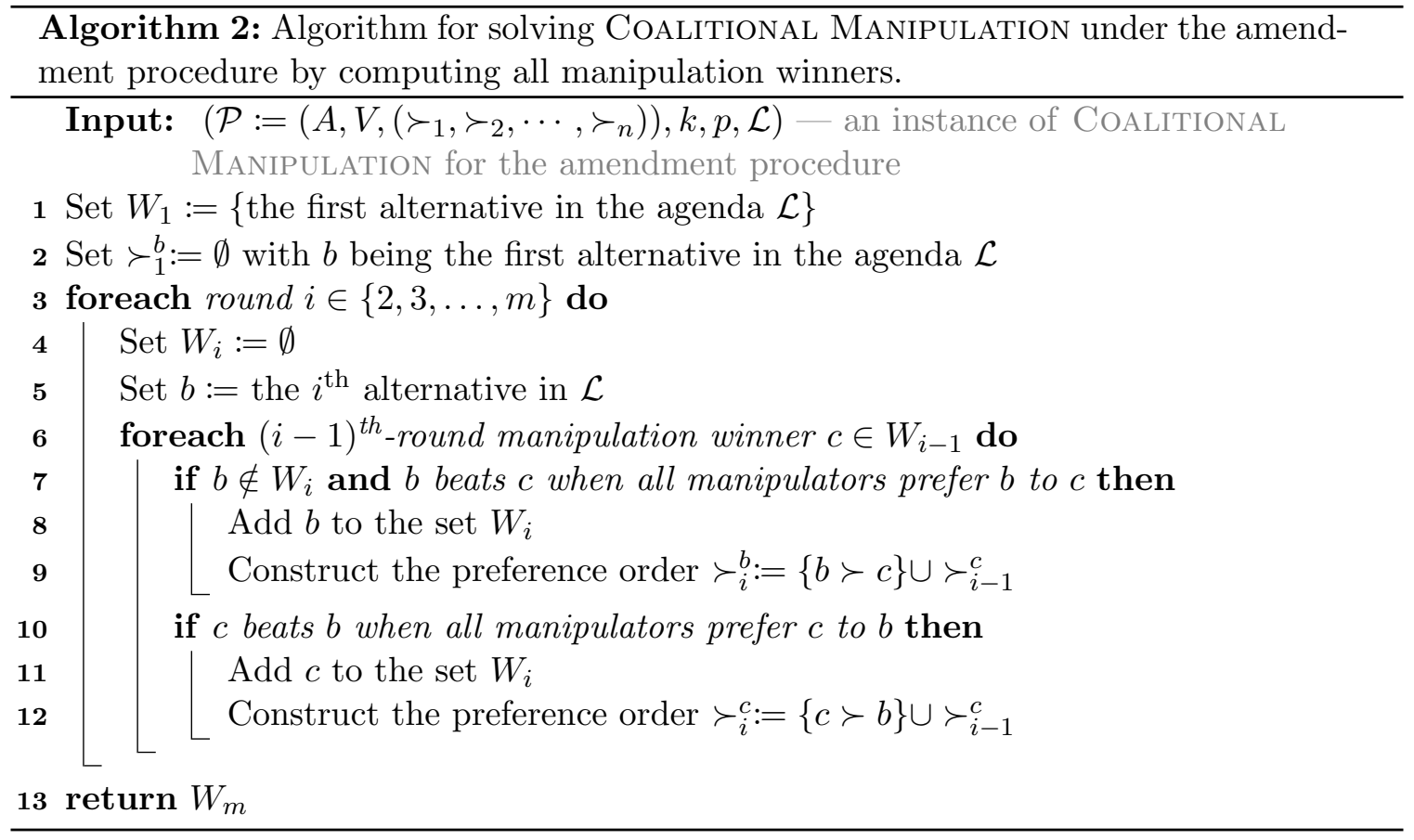

By Lemma 2, we can indeed compute in time quadratic in the input size all alternatives that each can become an amendment winner in some manipulated profile with a coalition of $k$ additional voters, and we can compute the corresponding coalition for each of these alternatives.

Theorem 4. For the amendment procedure, solving CoALitional MANIPULATION and for yes-instances constructing the preference orders for the manipulators can be done in $O(n$. $\left.m^{2}\right)$ time, where $n$ denotes the number of voters and $m$ denotes the number of alternatives.

Proof. Based on Lemma 2 we build a recursive algorithm which, for each $i^{\text {th }}$-round amendment winner, constructs a linear order over the first $i$ alternatives in $\mathcal{L}$ starting with $i:=1$.

We denote by $W_{i}$ the set of all $i^{\text {th }}$-round manipulation winners. For each $i^{\text {th }}$-round manipulation winner $c$, we denote by $\succ_{i}^{c}$ the preference orders over the first $i$ alternatives in the agenda $\mathcal{L}$ such that $c$ becomes an $i^{\text {th }}$-round amendment winner by adding $k$ manipulators with a preference order that extends $\succ_{i}^{c}$.

Algorithm 2 computes all alternatives that can become an amendment winner when a coalition of $k$ manipulators is added. Obviously, for the first round, the set $W_{1}$ and its corresponding preference order are computed correctly. By Lemma 2(1), we know that Steps (7)-(9) are correct, and by Lemma 2(2), we know that Steps (10)-(12) are correct. Since each alternative from $W_{m}$ is a last-round manipulation winner, we answer "yes" and return the corresponding preference order for the input the instance $\left(\mathcal{P}:=\left(A, V,\left(\succ_{1}, \succ_{2}\right.\right.\right.$ $\left.\left.\left., \cdots, \succ_{n}\right)\right), k, p, \mathcal{L}\right)$ if $p \in W_{m}$ and "no" otherwise.

As for the running time, first, we do some preprocessing: for each two distinct alternatives $b, c$, we check whether adding $k$ manipulators can make $b$ beat $c$; let the Boolean 
variable $T(b, c)$ have value one if this is the case and zero otherwise. Computing all these Boolean values runs in $O\left((k+n) \cdot m^{2}\right)$ time.

To compute $W_{i}, 2 \leq i \leq m$, we inspect $T(b, c)$ for every alternative $c$ in $W_{i-1}$ and for the $i^{\text {th }}$ alternative $b$ in the agenda $\mathcal{L}$. Thus computing all $W_{i}$ can be done in $O\left(m^{2}\right)$ time. The total running time is $O\left((n+k) \cdot m^{2}\right)$.

In Weighted Coalitional Manipulation, the voters of the coalition also come with integer weights, specified in binary. However, we have that the weighted and non-weighted cases are equivalent because of Observation 5. Observe that if the sum of the weights is greater than the number of the voters in the original profile, then there is always a successful manipulation. Thus, we can conclude the following.

Corollary 1. Solving Weighted Coalitional Manipulation and constructing the preference orders for the manipulators in a yes-instance can be solved in $O(n \cdot m)$ time for the successive procedure and in $O\left(n \cdot m^{2}\right)$ time for the amendment procedure, where $n$ denotes the number of voters and $m$ denotes the number of alternatives.

We close this section by the following observation that deciding whether a coalition of manipulators can achieve a better outcome is also polynomial-time solvable since we just need to solve CoAlitional Manipulation for every possible alternative that is preferred to the original winner by the manipulators:

Observation 6. Deciding whether a coalition of $k$ manipulators can achieve a better outcome by voting insincerely can be done in $O\left(n \cdot m^{2}\right)$ time for the successive procedure and in $O\left(n \cdot m^{3}\right)$ time for the amendment procedure, where $n$ denotes the number of voters and $m$ denotes the number of alternatives.

\section{Empirical Study of Agenda Control and Coalitional Manipulation}

The polynomial-time algorithms from Sections 3 and 4 allow us to efficiently check whether a control or a manipulation is successful, but do not tell us how often a successful control or manipulation can happen. In this section, we tackle this by investigating empirically how many alternatives can win through control (or manipulation). To this end, we use data from the PrefLib collection of preference profiles (Mattei \& Walsh, 2013) to examine the ratio of profiles that admit successful agenda control or manipulation operations. The preference profiles in PrefLib are not taken from parliamentary votes. Nevertheless, we decided to use PrefLib simply because it is one of the few free, online accessible libraries that we know of. One could extend our experiments once more data is available. Moreover, the voting procedures studied in this article are not restricted to parliamentary elections.

As we will see in the next section, since only one case of the Possible Winner and NeCESSARY WINNER is polynomial-time solvable and since PrefLib offers only a very restricted variant of incomplete preferences, we do not run experiments for POSSIBLE WinNer or Necessary Winner. Our algorithms for Agenda Control and Coalitional ManipuLATION are written in $\mathrm{C}++$ and are freely available through http://www.akt.tu-berlin. de/menue/software/. 


\subsection{Data Background}

PrefLib is a library for real-world preferences. The data is provided by various research groups. As of December 2016, PrefLib contained 315 profiles with complete preference orders: 100 of them have three alternatives, 108 of them have four alternatives, one of them has 6 alternatives, one of them has 7 alternatives, and the remaining 105 profiles have between 9 and 242 alternatives. Among all profiles with complete preference orders, 136 ones have an odd number of voters, where 56 of these have three alternatives, 52 of these have four alternatives, one of these has 6 alternatives, one of these has 7 alternatives, and the remaining 26 profiles have between 14 and 242 alternatives. The number of voters ranges from 5 to 14081 .

\subsection{Agenda Control}

For each of the 136 profiles with an odd number of voters (note that for reasons of simplicity, we only implemented our algorithms for odd numbers of voters), using the algorithms from Theorems 1 and 2, we compute the number $m_{s}$ (resp. $m_{a}$ ) of alternatives for which a successive (resp. amendment) agenda control is possible. Then, we calculate the control vulnerability ratio as

$$
\frac{m_{s}-1}{m-1} \text { and } \frac{m_{a}-1}{m-1}
$$

where $m$ denotes the number of alternatives. Note that we use $m-1$ because we factor out the alternative that wins originally. For instance, a control vulnerability ratio of 0.5 means that there are $\frac{m-1}{2}$ alternatives such that each of them can become the winner under an appropriately designed agenda.

We use the arithmetic mean and the geometric mean to compute the average values among all profiles (see Table 2). While the arithmetic mean is the sum of the control vulnerability ratios of all profiles divided by $t$, the geometric mean is the $t^{\text {th }}$ root of the product of the control vulnerability ratios of all profiles, where $t$ is the total number of profiles studied. We use the arithmetic mean because it is a commonly used standard mean and we use the geometric mean because it is suitable for comparing values of different kinds and the ratios we measure are indeed from profiles with different numbers of voters and alternatives.

\subsubsection{Evaluation on Agenda Control}

Our results show that the successive procedure tends to be more vulnerable to agenda control than the amendment procedure: For profiles with up to four alternatives, 0.157 of the alternatives have a chance to win under the successive procedure, while it is 0 under the amendment procedure. For the remaining profiles, the statistics are 0.099 versus 0.034 . We observe that no alternative other than the original winner can win under the amendment procedure by altering the agenda when the number of alternatives is at most four; there are one third of such profiles. This is remarkable since the values mean that the original winner of each of the one-third profiles is already a Condorcet winner. The values of the geometric mean are zero. This tells us that there is at least one profile from the PrefLib data for 


\begin{tabular}{|c|c|c|c|c|}
\hline \multirow{2}{*}{ control vulnerability ratio } & \multicolumn{2}{|c|}{$m \leq 4$} & \multicolumn{2}{|c|}{$m \geq 5$} \\
\hline & Successive & Amendment & Successive & Amendment \\
\hline Arithmetic mean & 0.157 & 0.000 & 0.099 & 0.034 \\
\hline Geometric mean & 0.000 & 0.000 & 0.000 & 0.000 \\
\hline
\end{tabular}

Table 2: Experiments on agenda control with PrefLib data. We evaluate all 136 profiles from PrefLib that have linear preference orders and an odd number of voters. We distinguish between profiles with $m \leq 4$ alternatives, and those with $m \geq 5$ alternatives. The reason for this separation is twofold. First, while a large number of profiles has either three or four alternatives (one third each), for $m \geq 5$, in most cases, less than five profiles have $m$ alternatives. Second, the results for profiles with up to four alternatives are very different from the results for the other profiles.

which there is a majority (and thus a Condorcet) winner, making the control vulnerability ratio to zero for the amendment procedure and the successive procedure.

\subsection{Manipulation}

Since PrefLib does not offer any agenda, we have to generate a set $\mathcal{Z}$ of agendas for manipulation to obtain a good representation. The size of $\mathcal{Z}$ depends on the number $m$ of alternatives:

1. If $m \leq 8$, then let $\mathcal{Z}$ be the set of all possible agendas, that is, $|\mathcal{Z}|:=m$ !.

2. Otherwise, we generate a set $\mathcal{Z}$ of agendas by randomly choosing $|\mathcal{Z}|:=\min \left(n^{2}, 8 !\right)$ agendas from the set of all possible agendas, where $n$ denotes the number of voters in the input. All possible agendas are equally likely of being chosen for $\mathcal{Z}$.

For each alternative $c$ and for each agenda $\mathcal{L} \in \mathcal{Z}$, using the algorithms behind Theorems 3 and 4 , we compute the minimum coalition size, that is, the minimum number of voters needed to make $c$ a successive (resp. an amendment) winner. Let this number for the successive (resp. amendment) procedure be $\kappa_{s}(\mathcal{P}, c, \mathcal{L})\left(\right.$ resp. $\left.\kappa_{a}(\mathcal{P}, c, \mathcal{L})\right)$. This number each time is upper-bounded by $n+1$, where $n$ denotes the number of voters in the given profile.

We calculate the manipulation resistance ratio as

$$
\frac{\sum_{\mathcal{L} \in \mathcal{Z}} \sum_{c \in C} \kappa_{s}(\mathcal{P}, c, \mathcal{L})}{|\mathcal{Z}| \cdot(m-1) \cdot(n+1)} \quad \text { and } \quad \frac{\sum_{\mathcal{L} \in \mathcal{Z}} \sum_{c \in C} \kappa_{a}(\mathcal{P}, c, \mathcal{L})}{|\mathcal{Z}| \cdot(m-1) \cdot(n+1)} .
$$

For instance, a manipulation resistance ratio of 0.4 means that each alternative in a profile needs a coalition of $0.4 \cdot(n+1)$ additional manipulators, on average, to become a winner.

From our experimental results, we observe that on average, a successful manipulation needs at least $n / 2$ manipulators (see the first row in Table 3 ), where $n$ denotes the number of voters in the given profile. This is because in order to become a winner, most alternatives need a coalition of at least $n+1$ additional voters. To get a more thorough understanding of the manipulation issue, however, we consider two related concepts: 


\begin{tabular}{lccccc}
\hline \multirow{2}{*}{ Measurements } & \multicolumn{2}{c}{$m \leq 8$} & & \multicolumn{2}{c}{$m \geq 9$} \\
\cline { 2 - 3 } \cline { 5 - 6 } & Successive & Amendment & & Successive & Amendment \\
\hline manipulation resistance ratio & 0.455 & 0.402 & & 0.945 & 0.924 \\
$2^{\text {nd }}$ winner coalition ratio & 0.288 & 0.222 & & 0.523 & 0.468 \\
smallest coalition ratio & 0.263 & 0.221 & & 0.386 & 0.385 \\
\hline
\end{tabular}

Table 3: Experiments on manipulation with real-world data. We evaluate all 314 profiles from PrefLib that have linear preference orders. We separately consider profiles with $m \leq 8$ and $m \geq 9$ alternatives. The reason for this separation is that we only consider all $m$ ! possible agendas when $m \leq 8$. As all three measures are ratios between $[0,1]$, we use the geometric mean to compute the average and omit showing the values using the arithmetic mean since they are similar to the ones obtained with the geometric mean.

- The ratio of the $2^{\text {nd }}$ winner coalition size, that is, the coalition size for the alternative that becomes a winner after the original winner is removed. Formally, the $2^{\text {nd }}$ winner coalition is defined as

$$
\frac{\sum_{\mathcal{L} \in \mathcal{Z}} \kappa_{s}\left(\mathcal{P}, c^{*}, \mathcal{L}\right)}{|\mathcal{Z}| \cdot(n+1)} \quad \text { and } \quad \frac{\sum_{\mathcal{L} \in \mathcal{Z}} \kappa_{a}\left(\mathcal{P}, c^{*}, \mathcal{L}\right)}{|\mathcal{Z}| \cdot(n+1)}
$$

where $c^{*}$ is a successive (resp. an amendment) winner after the original winner is removed.

- The ratio of the smallest coalition size, that is, the size $\sigma_{s}(\mathcal{P}, \mathcal{L})\left(\operatorname{resp} . \sigma_{a}(\mathcal{P}, \mathcal{L})\right)$ of the smallest coalition that makes some alternative other than the original winner win. Formally, the smallest coalition is defined as

$$
\frac{\sum_{\mathcal{L} \in \mathcal{Z}} \sigma_{s}(\mathcal{P}, \mathcal{L})}{|\mathcal{Z}| \cdot(n+1)} \quad \text { and } \quad \frac{\sum_{\mathcal{L} \in \mathcal{Z}} \sigma_{a}(\mathcal{P}, \mathcal{L})}{|\mathcal{Z}| \cdot(n+1)}
$$

\subsubsection{Evaluation on Manipulation}

Our results show that successful manipulations with few voters are rare; let $n$ denote the number of voters in the respective profile: For profiles with up to eight alternatives the average coalition size is at least $0.4 \cdot(n+1)$ (even the $2^{\text {nd }}$ winner coalition size is $0.2 \cdot(n+1)$; the smallest coalition size is only slightly lower), while for profiles with at least nine alternatives the average coalition size is almost $n+1$ (even the $2^{\text {nd }}$ winner coalition size is roughly $0.5 \cdot(n+1))$. Comparing the two procedures, it seems that making an alternative win under the successive procedure requires more manipulators than making it win under the amendment procedure. The difference, however, is not very pronounced.

\section{Possible/Necessary Winner}

We typically have partial knowledge about how the voters will vote, and about how the agenda will order the alternatives. Nevertheless, we might be interested in what may or 
may not be the final outcome. Does our favorite alternative stand any chance of winning? Is it inevitable that the government's alternative will win? Is there an agenda under which our alternative can win? Hence, we consider the computational question of which alternative possibly or necessarily wins.

Possible (resp. Necessary) Winner

Input: A preference profile $\mathcal{P}:=\left(A, V,\left(\succ_{1}, \succ_{2}, \cdots, \succ_{n}\right)\right)$, a preferred alternative $p \in$ $A$, and a partial agenda $\mathcal{B}$.

Question: Can $p$ win in a (resp. every) completion of the profile $\mathcal{P}$ for an (resp. every) agenda which completes $\mathcal{B}$ ?

We remark that in the definition of Possible Winner and Necessary Winner, the given agenda is a partial order. This is because we are rather interested in the computational complexity of determining winners under uncertainty. Our hardness results indicate however, that the problems remain hard even when the given agenda is already a linear order.

An upper bound for the computational complexity of both problems is easy to see: Possible Winner (resp. Necessary Winner) is contained in NP (resp. in coNP) for both the successive procedure and the amendment procedure, because one can determine a winner for both procedures in polynomial time and so just has to "guess the completion" in polynomial time. Thus, in order to show NP-completeness (resp. coNP-completeness), we only need to show NP-hardness (resp. coNP-hardness).

\subsection{Possible Winner}

Our first two results imply that as soon as the voters may have incomplete preference orders, deciding who may be a possible winner is NP-hard even if the agenda is already a linear order. Nevertheless, for the parameter "number $m$ of alternatives", we obtain that both problems are fixed-parameter tractable, meaning that there is an algorithm solving the problems in $f(m) \cdot|I|^{O(1)}$ time, where $f$ is a computable function only dependent on $m$ and $|I|$ is the size of an input instance for Possible Winner.

Theorem 5. For the successive procedure, POSSIBle Winner with the given agenda being linear is NP-complete.

Proof. We show the NP-hardness by reducing from the NP-complete IndePEndent SET problem in polynomial time.

\section{INDEPENDENT SET}

Input: An undirected graph $G:=(U, E)$ and a non-negative integer $h$.

Question: Is there an independent set of size at least $h$, that is, a subset of at least

$h$ vertices such that no two of them are adjacent to each other?

We will give a concrete example for the reduction right after the proof. Let $(G:=$ $(U, E), h)$ be an instance of Independent Set, where $U:=\left\{u_{1}, \ldots, u_{r}\right\}$ denotes the set of $r$ vertices and $E:=\left\{e_{1}, \ldots, e_{s}\right\}$ denotes the set of $s$ edges. We assume that $r \geq 3$ and $2 \leq h \leq r-1$. We construct a Possible Winner instance $((A, V), p, \mathcal{B})$ as follows. The 
set $A$ of alternatives consists of the preferred alternative $p$, one dummy alternative $d$, and of one edge alternative $c_{j}$ for each edge $e_{j} \in E$ :

$$
A:=\{p, d\} \cup\left\{c_{j} \mid e_{j} \in E\right\} .
$$

We construct three groups of voters, where only the first group of voters has partial orders while the remaining two groups have linear orders. We let $\overrightarrow{A \backslash\{p, d\}}$ denote the linear order $c_{1} \succ c_{2} \succ \cdots \succ c_{s}$.

1. For each vertex $u_{i} \in U$, construct a vertex voter $v_{i}$ with a partial preference order specified by

$$
v_{i}: \overrightarrow{I\left(u_{i}\right)} \succ p \succ \overrightarrow{A \backslash\left(\{p, d\} \cup I\left(u_{i}\right)\right)} \text { and } d \succ \overrightarrow{A \backslash\left(\{p, d\} \cup I\left(u_{i}\right)\right)} \text {, }
$$

where $I\left(u_{i}\right)$ denotes the set of edge alternatives corresponding to edges incident to vertex $u_{i}$.

Briefly put, voter $v_{i}$ prefers every "incident" edge alternative, $p$, and $d$ to all "nonincident" edge alternatives. He also prefers the incident edge alternatives to $p$ but thinks that $d$ is incomparable to $p$ and to every "incident" edge alternative.

2. Construct $h-2$ auxiliary voters with the same preference order:

$$
h-2 \text { voters : } \quad \overrightarrow{A \backslash\{p, d\}} \succ d \succ p .
$$

3. Construct another $r-h-1$ auxiliary voters with the same preference order:

$$
r-h-1 \text { voters : } \overrightarrow{A \backslash\{p, d\}} \succ p \succ d .
$$

We have constructed a total of $2 r-3$ voters. Thus, to be a majority winner, an alternative needs to be ranked at the first place by at least $r-1$ voters.

Let the agenda $\mathcal{B}$ be the linear order $c_{1} \triangleright c_{2} \triangleright \cdots \triangleright c_{s} \triangleright p \triangleright d$. This completes the construction, which can clearly be computed in polynomial time.

We show that $G$ has an independent set of size at least $h$ if and only if $p$ can possibly win under the successive procedure in the constructed profile.

For the "only if" part, assume that $G$ admits an independent set $U^{\prime} \subseteq U$ of size at least $h$. We complete the partial preference orders of the vertex voters as follows.

1. For each vertex $u_{i} \in U^{\prime}$, let voter $v_{i}$ have the preference order

$$
\overrightarrow{I\left(u_{i}\right)} \succ p \succ d \succ \overrightarrow{A \backslash\left(\{p, d\} \cup I\left(u_{i}\right)\right)} .
$$

2. For each vertex $u_{i} \in U \backslash U^{\prime}$, let voter $v_{i}$ have the preference order

$$
d \succ \overrightarrow{I\left(u_{i}\right)} \succ p \succ \overrightarrow{A \backslash\left(\{p, d\} \cup I\left(u_{i}\right)\right)} .
$$

Since $U^{\prime}$ is an independent set, every edge alternative is preferred to $d$ by at most one vertex voter. Together with the remaining $r-3$ auxiliary voters, every edge alternative is preferred to $d$ by at most $r-2$ voters, causing each edge alternative to be deleted if it is considered prior to $d$ in an agenda (note that we have $2 r-3$ voters). Hence, by our agenda, 
where all edge alternatives are in front of $d$, all edge alternatives are deleted. In the final round, since the independent set $U^{\prime}$ has size at least $h$, at least $h$ vertex voters prefer $p$ to $d$. Then, $p$ will beat $d$, because all $r-h-1$ auxiliary voters of the second group prefer $p$ to $d$, making $p$ a successive winner.

For the "if" part, assume that $p$ can possibly become a successive winner, which means that we can complete the vertex voters' preference orders, ensuring $p$ 's victory. Let $V^{\prime}$ be the set of vertex voters that prefer $p$ to $d$ in such a completion. Since a total of $r-h-1$ auxiliary voters prefer $p$ to $d$, in order to make $p$ beat $d$ in the last round, $V^{\prime}$ must have at least $h$ voters (note that the majority quota is $r-1$ ). We show that the vertex subset $U^{\prime}$ corresponding to $V^{\prime}$ is an independent set, that is, no two vertices in $U^{\prime}$ are adjacent. Suppose for the sake of contradiction that $U^{\prime}$ contains two adjacent vertices $u$ and $u^{\prime}$; denote the edge $\left\{u, u^{\prime}\right\}$ by $e_{j}$. In the completed profile, since $p$ is a successive winner, there must be a round where the corresponding edge alternative $c_{j}$ is considered. Since all edge alternatives with a lower index have been deleted already, by construction of the preference orders, a total of $r-1$ voters (the two vertex voters corresponding to $u, u^{\prime}$ and all $r-3$ auxiliary voters) rank $c_{j}$ in the first position in the profile restricted to the alternatives $c_{j}, c_{j+1}, \ldots, c_{s}, p, d$; note that all voters prefer $c_{\ell} \succ c_{\ell+1}, 1 \leq \ell \leq s-1$. This, however, will make $c_{j}$ win-a contradiction.

We illustrate the NP-hardness reduction for the proof of Theorem 5 through an example. Figure 1(a) depicts an undirected graph $G$ with six vertices and eight edges. We set $h:=4$. The gray vertices form an independent set of size four. Figure 1(b) depicts the instance for Possible Winner under the successive procedure constructed by the reduction. The included profile has $6+(4-2)+(6-4-1)=9$ voters and $2+8=10$ alternatives. By completing this profile according to our proof, we can verify that $p$ is a successive winner.

Now, we show that Possible Winner remains NP-complete for the amendment procedure. As already discussed, containment in NP is obvious.

Theorem 6. For the amendment procedure, POSSIBLE WINNER with the given agenda being linear is NP-complete.

Proof. We show NP-hardness by reducing from the NP-complete VERTEx Cover problem in polynomial time:

\section{VerTex COVER}

Input: An undirected graph $G=(U, E)$ and an integer $k \leq|U|$.

Question: Is there a vertex cover $U^{\prime} \subseteq U$ of at most $k$ vertices, that is, $\left|U^{\prime}\right| \leq k$ and

$\forall e \in E: e \cap U^{\prime} \neq \emptyset$ ?

We will present an example for the reduction right after the proof.

Let $(G=(U, E), h)$ be a Vertex Cover instance, where $U:=\left\{u_{1}, \ldots, u_{r}\right\}$ denotes the set of vertices and $E:=\left\{e_{1}, \ldots, e_{s}\right\}$ denotes the set of edges. We assume that $r \geq 1$ and $1 \leq h \leq r-1$.

We construct a Possible Winner instance $\left(\left(A, V,\left(\succ_{1}, \succ_{2}, \cdots, \succ_{n}\right)\right), p, \mathcal{B}\right)$ as follows. The set $A$ of alternatives consists of the preferred alternative $p$, one helper alternative $b$, one dummy alternative $d$, and one edge alternative $c_{j}$ for each edge $e_{j} \in E$ :

$$
A:=\{p, b, d\} \cup\left\{c_{j} \mid e_{j} \in E\right\} .
$$




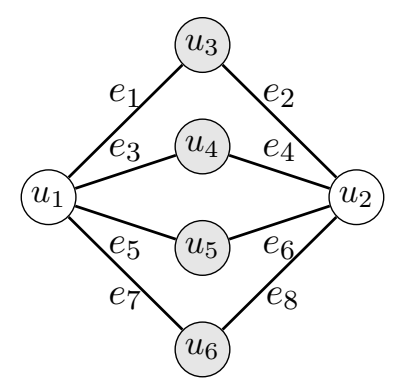

(a)
Voter $v_{1}: \quad\left\{c_{1} \succ c_{3} \succ c_{5} \succ c_{7} \succ p, d\right\} \succ c_{2} \succ c_{4} \succ c_{6} \succ c_{8}$

Voter $v_{2}: \quad\left\{c_{2} \succ c_{4} \succ c_{6} \succ c_{8} \succ p, d\right\} \succ c_{1} \succ c_{3} \succ c_{5} \succ c_{7}$

Voter $v_{3}: \quad\left\{c_{1} \succ c_{2} \succ p, d\right\} \succ c_{3} \succ c_{4} \succ c_{5} \succ c_{6} \succ c_{7} \succ c_{8}$

Voter $v_{4}: \quad\left\{c_{3} \succ c_{4} \succ p, d\right\} \succ c_{1} \succ c_{2} \succ c_{5} \succ c_{6} \succ c_{7} \succ c_{8}$

Voter $v_{5}: \quad\left\{c_{5} \succ c_{6} \succ p, d\right\} \succ c_{1} \succ c_{2} \succ c_{3} \succ c_{4} \succ c_{7} \succ c_{8}$

Voter $v_{6}: \quad\left\{c_{7} \succ c_{8} \succ p, d\right\} \succ c_{1} \succ c_{2} \succ c_{3} \succ c_{4} \succ c_{5} \succ c_{6}$

Two voters: $c_{1} \succ c_{2} \succ c_{3} \succ c_{4} \succ c_{5} \succ c_{6} \succ c_{7} \succ c_{8} \succ d \succ p$

One voter: $\quad c_{1} \succ c_{2} \succ c_{3} \succ c_{4} \succ c_{5} \succ c_{6} \succ c_{7} \succ c_{8} \succ p \succ d$

(b)

Figure 1: Illustration for the proof of Theorem 5. (a) An undirected graph with six vertices and eight edges. The graph has an independent set of size four (the gray vertices) and has a vertex cover of size two (the white vertices). (b) The instance $\left(\left(A, V,\left(\succ_{1}, \succ_{2}, \cdots, \succ_{n}\right)\right), p, \mathcal{B}\right)$ of the Possible Winner problem for the successive procedure obtained from the VerTex Cover-instance depicted on the left with $h=4$, where $A:=\left\{c_{1}, c_{2}, c_{3}, c_{4}, c_{5}, c_{6}, c_{7}, c_{8}, p, d\right\}$, the voter set $V$ and the corresponding preference orders and the agenda are depicted in the figure. From the constructed agenda, we notice that in order to let $p$ beat $d$ in the last two rounds, at least $h=4$ vertex voters must rank $p \succ d$, but in order not to let an edge alternative obtain too much "support", at most one of its "incident" vertex voters should rank $p$ higher than $d$.

We construct three groups of voters for the voter set $V$, where only the first group of voters has partial preference orders while the remaining two groups of voters have linear preference orders. Let $\overrightarrow{A \backslash\{p, b, d\}}$ denote the order $c_{1} \succ c_{2} \succ \ldots \succ c_{s}$.

1. For each vertex $u_{i}$, construct a vertex voter $v_{i}$ with partial order specified by

$$
v_{i}: \overrightarrow{A \backslash\left(\{p, b, d\} \cup I\left(u_{i}\right)\right)} \succ b \succ p \text { and } \overrightarrow{A \backslash\left(\{p, b, d\} \cup I\left(u_{i}\right)\right)} \succ \overrightarrow{I\left(u_{i}\right)} \succ d,
$$

where $\overrightarrow{A \backslash\left(\{p, b, d\} \cup I\left(u_{i}\right)\right)}$ denotes the order derived from $\overrightarrow{A \backslash\{p, b, d\}}$ by removing $u_{i}$ 's "incident" edge alternatives.

Briefly put, voter $v_{i}$ prefers every "non-incident" edge alternative to the remaining ones. He prefers $b$ to $p$ and prefers every "incident" edge alternative to $d$. But, he thinks $b$ and $p$ are incomparable to all "incident" edge alternatives and to $d$.

2. Construct $r-h-1$ auxiliary voters with the same order:

$$
r-h-1 \text { voters : } p \succ \overrightarrow{A \backslash\{p, b, d\}} \succ b \succ d .
$$

3. Finally, construct another $h$ auxiliary voters with the same order:

$$
h \text { voters : } \quad p \succ d \succ \overrightarrow{A \backslash\{p, b, d\}} \succ b .
$$

Note that we have constructed a total of $2 r-1$ voters. Thus, an alternative $a$ beats another alternative $a^{\prime}$ if and only if at least $r$ voters prefer $a$ to $a^{\prime}$. 

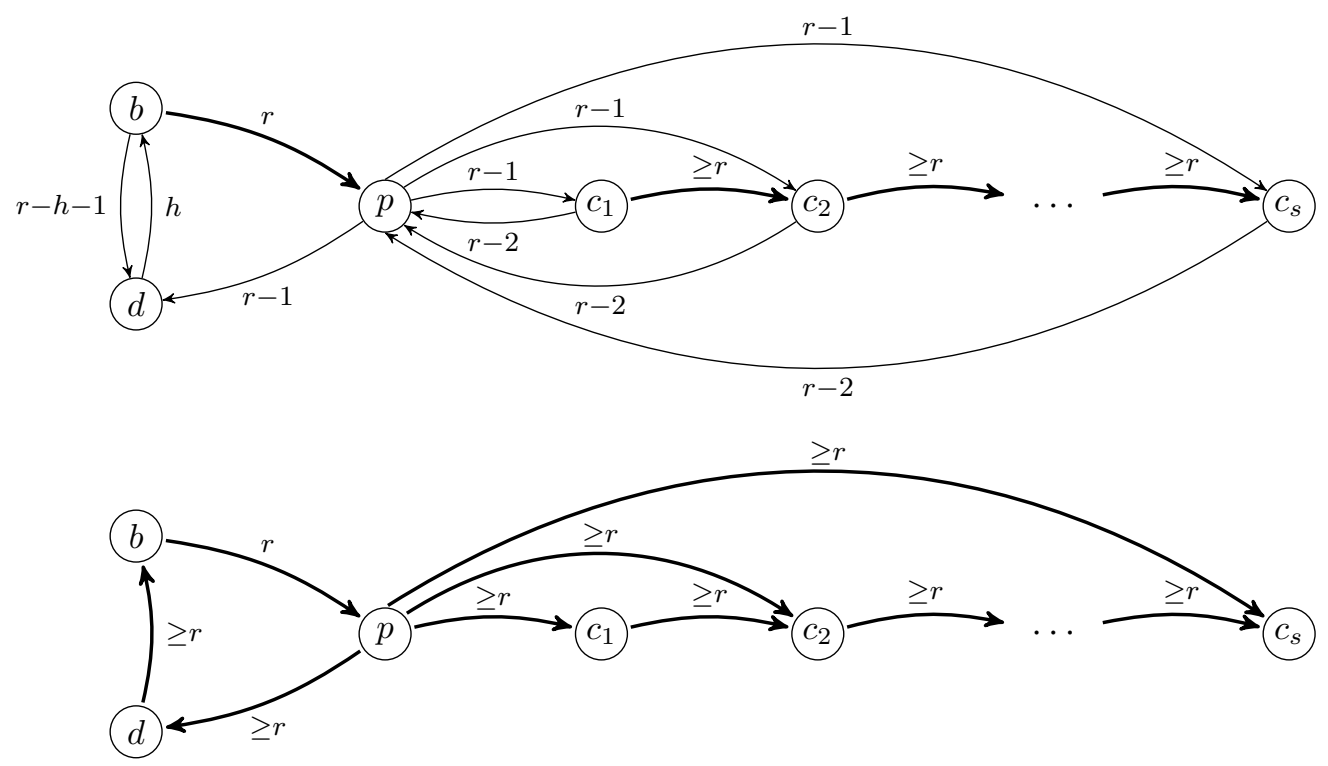

Figure 2: Top: The weighted majority graph (not including all arcs) for the obtained profile with $2 r-1$ voters and $s+3$ alternatives in the reduction for POSSIBLE Winner under the amendment procedure (Theorem 6). In particular, for each two vertices $c$ and $d$, if there is an arc from $c$ to $d$ with weight more than $r$ (that is, a strict majority of voters prefer the alternative corresponding to $c$ to the alternative corresponding to $d$ ), then we omit the back-arc from $d$ to $c$. The agenda is $b \triangleright d \triangleright p \triangleright c_{s} \triangleright c_{s-1} \triangleright \cdots \triangleright c_{1}$. We use the same symbol for both the alternative and its corresponding vertex. For instance, there is an arc from $b$ to $p$ with weight $r$ because exactly $r$ voters prefer $b$ to $p$. They all come from the first group of voters. We draw an arc $\left(a, a^{\prime}\right)$ as a thick line if there is already a majority of voters preferring $a$ to $a^{\prime}$. For the sake of brevity, some (irrelevant) arcs are omitted. By "..." we refer to the remaining edge alternatives $c_{3}, c_{4}, \ldots, c_{s-1}$, in increasing order. Bottom: The weighted majority graph (not including all arcs) for the completed profile corresponding to a vertex cover. All remarks for the figure on the top equally apply to the figure at the bottom.

Let the agenda $\mathcal{B}$ be the linear order $b \triangleright d \triangleright p \triangleright c_{s} \triangleright c_{s-1} \triangleright \cdots \triangleright c_{1}$. This completes the construction, which can clearly be computed in polynomial time.

We illustrate the corresponding majority graph for the constructed profile in Figure 2.

Now, we show that the two instances $(G, h)$ and $\left(\left(A, V,\left(\succ_{1}, \succ_{2}, \cdots, \succ_{n}\right)\right), p, \mathcal{B}\right)$ are equivalent, that is, $G$ has a vertex cover of size at most $h$ if and only if $p$ can possibly win under the amendment procedure.

For the "only if" part, assume that set $U^{\prime}$ is a vertex cover of size at most $h$. We complete the partial orders of the vertex voters as follows.

1. For each vertex $u_{i} \in U^{\prime}$, let $v_{i}$ have the preference order

$$
\overrightarrow{A \backslash\left(\{p, b, d\} \cup I\left(u_{i}\right)\right)} \succ b \succ p \succ \overrightarrow{I\left(u_{i}\right)} \succ d .
$$




\begin{tabular}{ll} 
Voter $v_{1}:$ & $c_{2} \succ c_{4} \succ c_{6} \succ c_{8} \succ\left\{c_{1} \succ c_{3} \succ c_{5} \succ c_{7} \succ d, b \succ p\right\}$ \\
Voter $v_{2}:$ & $c_{1} \succ c_{3} \succ c_{5} \succ c_{7} \succ\left\{c_{2} \succ c_{4} \succ c_{6} \succ c_{8} \succ d, b \succ p\right\}$ \\
Voter $v_{3}:$ & $c_{3} \succ c_{4} \succ c_{5} \succ c_{6} \succ c_{7} \succ c_{8} \succ\left\{c_{1} \succ c_{2} \succ d, b \succ p\right\}$ \\
Voter $v_{4}:$ & $c_{1} \succ c_{2} \succ c_{5} \succ c_{6} \succ c_{7} \succ c_{8} \succ\left\{c_{3} \succ c_{4} \succ d, b \succ p\right\}$ \\
Voter $v_{5}:$ & $c_{1} \succ c_{2} \succ c_{3} \succ c_{4} \succ c_{7} \succ c_{8} \succ\left\{c_{5} \succ c_{6} \succ d, b \succ p\right\}$ \\
Voter $v_{6}:$ & $c_{1} \succ c_{2} \succ c_{3} \succ c_{4} \succ c_{5} \succ c_{6} \succ\left\{c_{7} \succ c_{8} \succ d, b \succ p\right\}$ \\
Three voters: & $p \succ c_{1} \succ c_{2} \succ c_{3} \succ c_{4} \succ c_{5} \succ c_{6} \succ c_{7} \succ c_{8} \succ b \succ d$ \\
Two voters: & $p \succ d \succ c_{1} \succ c_{2} \succ c_{3} \succ c_{4} \succ c_{5} \succ c_{6} \succ c_{7} \succ c_{8} \succ b$ \\
\hline Agenda $\mathcal{B}:$ & $b \triangleright d \triangleright p \triangleright c_{8} \triangleright c_{7} \triangleright c_{6} \triangleright c_{5} \triangleright c_{4} \triangleright c_{3} \triangleright c_{2} \triangleright c_{1}$
\end{tabular}

Table 4: The instance $\left(\left(A, V,\left(\succ_{1}, \succ_{2}, \cdots, \succ_{n}\right)\right), p, \mathcal{B}\right)$ of Possible Winner for the amendment procedure where $V:=\left\{v_{1}, v_{2}, \ldots, v_{6}\right\}$ and $A:=\left\{c_{1}, c_{2}, \ldots, c_{8}, p, d\right\}$, obtained from the graph in Figure 1(a) and $h=2$, the voter set $V$ and the corresponding preference orders and the agenda are depicted above. By the construction of the agenda, we notice that in order to let $p$ become an amendment winner, at most $h=2$ vertex voters can $\operatorname{rank} b \succ p \succ c_{j} \succ d$ for some $c_{j}$. In order to beat every edge alternative $c_{j}$, at least one "incident" voter must rank $b \succ p \succ c_{j}$.

2. For each vertex $u_{i} \in U \backslash U^{\prime}$, let $v_{i}$ have the preference order

$$
\overrightarrow{A \backslash\left(\{p, b, d\} \cup I\left(u_{i}\right)\right)} \succ \overrightarrow{I\left(u_{i}\right)} \succ d \succ b \succ p .
$$

Since $\left|U \backslash U^{\prime}\right| \geq r-h$, at least $r-h$ vertex voters prefer $d$ to $b$. Furthermore, all $h$ voters from the third group prefer $d$ to $b$, implying that a strict majority of voters prefer $d$ to $b$. Thus, alternative $d$ beats $b$ and survives as the second round winner. Since we assume that $h \geq 1$, at least one additional vertex voter prefers $p$ to $d$, and since all $r-1$ voters from the second and the third group prefer $p$ to $d$, we obtain that alternative $p$ beats $d$ and survives as the third round winner. Since $U^{\prime}$ is a vertex cover, for each edge alternative $c_{j}, 1 \leq j \leq s$, there is at least one vertex voter preferring $p$ to $c_{j}$. This implies that $p$ beats $c_{j}$ (note that all $r-1$ auxiliary voters prefer $p$ to $c_{j}$ ), making $p$ an amendment winner.

For the "if" part, assume that there is a completion of the constructed profile, completing the preference orders of the vertex voters, so that $p$ is an amendment winner. Let $\mathcal{P}$ be such a completion and let $V^{\prime}$ be the set of all vertex voters who prefer $p$ to some edge alternative $c_{j}$. As we already noticed, since $p$ is in front of every edge alternative in the agenda, $p$ must beat every edge alternative. Thus, for each edge alternative $c_{j}$, there is at least one vertex voter $v_{i} \in V^{\prime}$ who prefers $p$ to $c_{j}$. By the construction of the preference orders, this means that $V^{\prime}$ corresponds to a vertex cover.

Every vertex voter in $V^{\prime}$ also prefers $b$ to $d$ (because he originally ranks $b \succ p$ and $c_{j} \succ d$ ). Since all $r-h-1$ auxiliary voters from the second group prefer $b$ to $d$ and since $p$ can only possibly win if $b$ does not survive the second round (because $b$ beats $p$ ), there are at most $h$ voters in $V^{\prime}$. This further implies that the vertex set corresponding to $V^{\prime}$ is a vertex cover of size at most $h$.

We illustrate the NP-hardness reduction through an example. Let us consider the undirected graph depicted in Figure 1(a) again. We set $h:=2$, and the vertex cover consists of 
the white vertices. Then, the constructed instance of Possible Winner for the amendment procedure has $6+(6-2-1)+2=11$ voters and $3+8=11$ alternatives. This instance can be found in Table 4 .

We have just shown that it is NP-hard to decide whether a given alternative can possibly be a successive (or amendment) winner, even when the given agenda is already a linear order (see Theorems 5 and 6 ). In both NP-hardness reductions, the number $m$ of alternatives and the number $n$ of voters are unbounded. In most parliamentary elections, however, there is only a limited number of alternatives. For this case we obtain tractability results. Specifically, we show that Possible Winner can be solved in $f(m) \cdot|I|^{O(1)}$ time (known as FPT-time, where FPT stands for fixed-parameter tractability), where $f$ is a computable function only dependent on $m$ and $|I|$ denotes the size of an input instance.

Our approach is based on an FPT-time reduction to the following problem.

Integer Linear Programming Feasibility

Input: An integer $m^{\prime} \times n^{\prime}$-matrix $A \in \mathbb{Z}^{m^{\prime} \times n^{\prime}}$ and an integer vector $b \in \mathbb{Z}^{m^{\prime}}$.

Question: Is there an integer vector $x \in \mathbb{Z}^{n^{\prime}}$ with $A \cdot x \leq b$ ?

The search variant of Integer Linear Programming Feasibility aims at finding an integer vector $x$ of $n^{\prime}$ variables fulfilling the constraints indicated by the constraint matrix $A$ and the goal vector $b$, that is, $A \cdot x \leq b$. Despite the NP-hardness, a famous algorithm by Lenstra (1983) shows that any InTEger Linear Programming FEASIBILITy instance with an $m^{\prime} \times n^{\prime}$-matrix $A$ and goal vector $b$ can be solved in $f\left(n^{\prime}\right) \cdot|(A, b)|^{O(1)}$ time, where $f$ is a function solely dependent on $n^{\prime}$, and $|(A, b)|$ denotes the number of bits in the binary representation of $A$ and $b$. In other words, Lenstra's result directly implies fixed-parameter tractability for the parameter "number $n^{\prime}$ of variables". ${ }^{2}$

We reduce Possible Winner to Integer Linear Programming Feasibility where the input matrix has $2^{O\left(m^{2}\right)}$ columns (that is, variables) and $2^{O\left(m^{2}\right)}+O(n)$ rows (that is, constraints), and the absolute value of each coefficient and of each entry in the goal vector is at most $2 n$ (recall that $m$ denotes the number of alternatives and $n$ the number of voters). By Lenstra (1983), Kannan (1987), Frank and Tardos (1987) this immediately implies fixed-parameter tractability for the parameter $m$, because the number of variables is upper-bounded by a function in $m$ and the number of bits needed to represent the ILP instance is upper-bounded by $2^{O\left(m^{2}\right)} \cdot\left(2^{O\left(m^{2}\right)}+n\right) \cdot \log (2 n)$.

Theorem 7. Let $m$ denote the number of alternatives and $n$ the number of voters of a given Possible Winner instance. Let ilp $\left(\rho_{1}, \rho_{2}, \rho_{3}\right)$ denote the running time of an algorithm solving an InTEger LineAR PROGRAMming FEASIBILITy instance with $\rho_{1}$ variables and $\rho_{2}$ constraints where the maximum of the absolute values of the coefficients and the constant terms is $\rho_{3}$, with $\rho_{1}, \rho_{2}, \rho_{3} \in \mathbb{N}$. Then, PossiBle WinneR can be solved in $O\left(m ! \cdot \operatorname{ilp}\left(m ! \cdot 2^{m^{2}}, 2 \cdot m ! \cdot 2^{m^{2}}+n, n\right)\right)$ time for the successive procedure and in $O\left(m ! \cdot 2^{m} \cdot \operatorname{ilp}\left(2^{m^{2}} \cdot m !, 2 \cdot n \cdot m !+m, 2 n\right)\right)$ time for the amendment procedure.

Proof. The general idea for both procedures is to "guess" the possible completion of the agenda as well as the sequential outcome of the voting regarding this agenda for which our

2. Kannan (1987) and Frank and Tardos (1987) improved the FPT running time to $O\left(\left(n^{\prime}\right)^{2.5 \cdot n^{\prime}+o\left(n^{\prime}\right)}\right.$. $|(A, b)|)$, which is currently the best known FPT running time for this setting. 
preferred $p$ may win and use integer linear programs (ILPs) to check whether such a guess is valid.

We introduce some notation for the description of our ILPs for both procedures. Let $\Pi$ denote the set of partial preference orders of the voters in the given profile. For each partial order $\succ \in \Pi$, we write $N(\succ)$ to denote the number of voters with partial order $\succ$ in the original profile, and we write $C(\succ)$ to denote the set of all possible linear orders completing $\succ$. For instance, if we have three alternatives $a, b, c$, then $C(\{a, b\} \succ c)=\{a \succ$ $b \succ c, b \succ a \succ c\}$ and $C(a \succ b \succ c)=\{a \succ b \succ c\}$. Accordingly, for each linear order $\succ^{*}$, we write $C^{-1}\left(\succ^{*}\right)$ to denote the set of all partial orders that $\succ^{*}$ can complete.

For each partial order $\succ$ and each possible completion $\succ^{*} \in C(\succ)$ of $\succ$, we introduce an integer variable $x\left(\succ, \succ^{*}\right)$ denoting the number of voters with partial order $\succ$ in the input profile that will have linear order $\succ^{*}$ in a possible completing profile. Since, for a set of $m$ elements, there are at most $2^{m^{2}}$ possible partial orders and at most $m$ ! possible linear orders, we have introduced at most $m ! \cdot 2^{m^{2}}$ variables. We use these variables for both the successive and the amendment procedures.

Successive procedure. Suppose we guess that our preferred alternative $p$ will possibly win under agenda $\mathcal{L}: a_{1} \triangleright a_{2} \triangleright \cdots \triangleright a_{y} \triangleright \cdots \triangleright a_{m}$ with $a_{y}=p$. We need one more notation: For each alternative $a_{i}, 1 \leq i \leq m-1$, let $F\left(a_{i}\right)$ denote the set of linear orders $\succ$ with $a_{i} \succ\left\{a_{i+1}, a_{i+2}, \ldots, a_{m}\right\}$. Now, the crucial point is that our preferred alternative $p$ is a successive winner under agenda $\mathcal{L}$ if and only if the following two conditions hold.

1. The profile can be completed so that no alternative $a_{i}, 1 \leq i \leq y-1$, is an $\left\{a_{i}, a_{i+1}, \ldots, a_{m}\right\}$-majority winner.

2. Alternative $p$ is an $\left\{a_{y}, a_{y+1}, \ldots, a_{m}\right\}$-majority winner.

We can describe these two conditions via a formulation of the ILP feasibility problem.

$$
\begin{array}{rlrl}
\sum_{\substack{\succ^{*} \in F(p) \\
\succ \in C^{-1}\left(\succ^{*}\right)}} x\left(\succ, \succ^{*}\right)>\frac{n}{2}, & \\
\sum_{\succ^{*} \in C(\succ)} x\left(\succ, \succ^{*}\right)=N(\succ), & & \forall \text { partial orders } \succ \in \Pi \text { and } \\
& \forall \text { linear completions } \succ^{*} \text { of } \succ, \\
\sum_{\succ^{*} \in F\left(a_{i}\right)} x\left(\succ, \succ^{*}\right) \leq \frac{n}{2}, & & \forall \text { integer } i \text { with } 1 \leq i \leq y-1, \\
\succ^{-1}\left(\succ^{*}\right) & & \\
x\left(\succ, \succ^{*}\right) \geq 0, & & \forall \text { partial orders } \succ \in \Pi \text { and } \\
\end{array}
$$

We explain the meaning of these three groups of constraints. First, constraint set (1) ensures that our preferred alternative $p$ is an $\left\{a_{y}, a_{y+1}, \ldots, a_{m}\right\}$-majority winner (condition 2) and constraint set (3) ensures that no alternative $a_{j}, 1 \leq j \leq y-1$, is an $\left\{a_{j}, a_{j+1}, \ldots, a_{m}\right\}$ majority winner (Condition 1). Second, constraint set (4) ensures that our variables are 
non-negative. Finally, constraint set (2) ensures that each voter's partial order is completed to exactly one linear order. The correctness of our formulation thus follows.

Before we come to the running time, let us mention again that we need to change the equations (2) and the strict inequality (1) to obtain an instance of INTEGER LINEAR PROGRAmming FEASIBILITy. This is quite straightforward and the numbers of additional variables and additional constraints are upper-bounded by the number of original constraints, respectively.

We derive our desired running time from the time of running an ILP. First of all, we brute-force search into all $m$ ! possible completions of the input agenda. Then, for each of these completions, we run an ILP with at most $m ! \cdot 2^{m^{2}}$ variables $x\left(\succ, \succ^{*}\right)$ and at most $2 \cdot n \cdot 2^{m^{2}}+m$ constraints where the absolute value of each coefficient and the constant term is at most $n$.

Amendment procedure. Just as for the successive procedure, we guess a completion of the agenda. Let $\mathcal{L}:=a_{1} \triangleright a_{2} \triangleright \cdots \triangleright a_{y} \triangleright \cdots \triangleright a_{m}$ be the guessed agenda with $a_{y}$ being our preferred alternative $p$. Apart from this, we also guess the amendment winner $b_{i}$ of each round $i$. We do this by guessing whether $a_{i}$ will be the $i^{\text {th }}$-round amendment winner: Let $b_{1}:=a_{1}$ because by definition, the first round amendment winner is $a_{1}$. For each $2 \leq i \leq y-1$, we guess a Boolean value $c_{i} \in\{0,1\}$, and let $b_{i}:=a_{i}$ if $c_{i}=1$ and let $b_{i}:=b_{i-1}$ otherwise. Furthermore, we set $c_{y}:=1$ since $b_{y}$ must be $a_{y}=p$. Note that guessing the amendment winner of a round numbered higher than or equal to $y$ is not necessary since the goal includes making $p$ beat every alternative $a_{z}$ with $z>y$.

Now, we can use an ILP formulation to check whether a valid guess can be realized by completing our partial profile. To this end, for each two distinct alternatives $a$ and $b$, let $G(a, b)$ denote the set of all linear orders with $a \succ b$. Our ILP formulation consists of four groups of constraints:

$$
\begin{aligned}
& \sum_{\succ^{*} \in C(\succ)} x\left(\succ, \succ^{*}\right)=N(\succ), \quad \begin{array}{l}
\text { partial orders } \succ \in \Pi \\
\text { and } \forall \text { linear }
\end{array} \\
& \text { completions } \succ^{*} \text { of } \succ \text {, }
\end{aligned}
$$

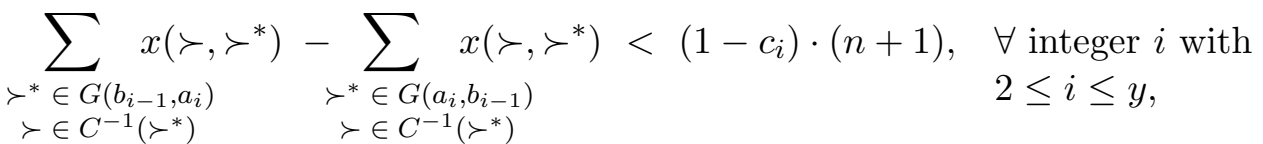

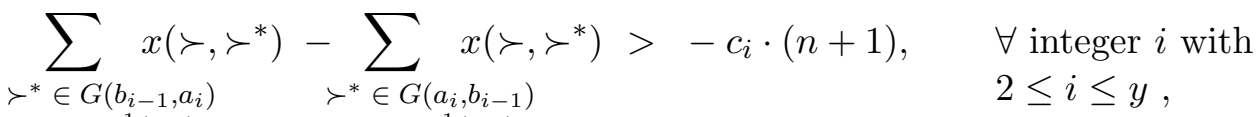

$$
\begin{aligned}
& \succ \in C^{-1}\left(\succ^{*}\right) \quad \succ \in C^{-1}\left(\succ^{*}\right)
\end{aligned}
$$

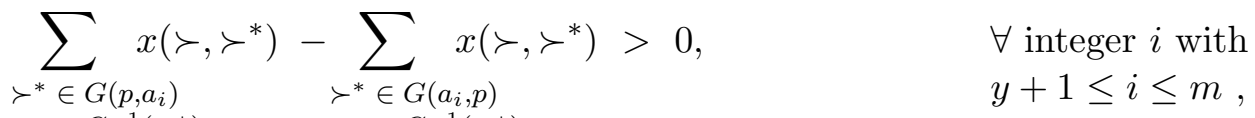

$$
\begin{aligned}
& \succ \in C^{-1}\left(\succ^{*}\right) \quad \succ \in C^{-1}\left(\succ^{*}\right) \\
& x\left(\succ, \succ^{*}\right) \geq 0
\end{aligned}
$$

The meaning of these four groups of constraints is as follows: The first group, Constraint (5), ensures that each voter's partial order is completed to exactly one linear order. 
The second and the third groups of constraints make the guessed alternative $b_{i}$ an $i^{\text {th }}$-round amendment winner: If $c_{i}=1$, then the right-hand side of Constraint (6) is zero. Hence, satisfying this constraint makes $a_{i}$ beat $b_{i-1}$ (because the number of voters preferring $a_{i}$ to $b_{i-1}$ is greater than the number of voters preferring $b_{i-1}$ to $a_{i}$ ). Thus, $b_{i}=a_{i}$. Otherwise, $c_{i}$ equals 0 , which implies that the right-hand side of Constraint (7) is zero. Hence, satisfying this constraint makes $b_{i-1}$ beat $a_{i}$ (because the number of voters preferring $b_{i-1}$ to $a_{i}$ is greater than the number of voters preferring $a_{i}$ to $b_{i-1}$ ). Consequently, the $i^{\text {th }}$ round amendment winner $b_{i}$ is $b_{i-1}$. The fourth group, Constraint (8), ensures that for each $i \in\{y+1, y+2, \ldots, m\}$, the number of voters preferring $p$ to $a_{i}$ is greater than the number of voters preferring $a_{i}$ to $p$, ensuring that $p$ beats $a_{i}$. The last group, Constraint (9), ensures that our variables are non-negative. The correctness of our ILP formulation thus follows.

To analyze the running time, first of all, for each valid completion of the agenda and valid sequence of the amendment winners (there are $m ! \cdot 2^{m}$ many), we run our ILP with at most $m ! \cdot 2^{m^{2}}$ variables $x\left(\succ, \succ^{*}\right)$ and with $O\left(2 \cdot m ! \cdot 2^{m^{2}}+2 n+m\right)$ constraints where the absolute value of each coefficient and each constant term is at most $2 n$.

Using the famous result of Lenstra (1983) (later improved by Kannan, 1987 and Frank \& Tardos, 1987), which states that the feasibility problem of an integer linear program can be solved in $O\left(\rho_{1}^{2.5 \rho_{1}+o\left(\rho_{1}\right)} \cdot|I|\right)$ time where $\rho_{1}$ denotes the number variables and $|I|$ the size of the integer linear program, we can derive the following tractability result because an integer linear program with $\rho_{1}$ variables and $\rho_{2}$ constraints, and whose coefficients and constant terms are between $-\rho_{3}$ and $\rho_{3}$, has $O\left(\rho_{1} \cdot \rho_{2} \cdot \log \left(\rho_{3}+2\right)\right)$ input bits.

Corollary 2. Let $m$ denote the number of alternatives and $n$ denote the number of voters of a given Possible WINNER instance. Then, for the successive procedure and the amendment procedure, Possible Winner can be solved in $O\left(\rho^{2.5 \rho+o(\rho)+2} \cdot \log (n+2)\right)$ time, where $\rho=$ $m ! \cdot 2^{m^{2}}$.

We close this section by remarking that the complexity result in Corollary 2 is of classification nature only. It would be interesting to know whether the fixed-parameter tractability results achieved through integer linear programming can also be achieved by a direct combinatorial (fixed-parameter) algorithm (also see the more general discussion of Bredereck et al., 2014, Key question 1).

\subsection{Necessary Winner}

In notable contrast to Possible Winner, Necessary Winner for the successive procedure tends to be computationally easier than for the amendment procedure. Observe that a given alternative $p$ is not a necessary winner if and only if the given profile and partial agenda can be completed so that there is another alternative $c \neq p$ such that one the following holds:

1. $c$ beats $p$ and it is considered after $p$ in the completed agenda or

2. $c$ is already a majority winner when considered and it is considered before $p$. 
The reason for the difference in complexity between the two voting procedures seems to be that for the successive procedure, checking whether there is a completion of the profile and the agenda satisfying one of the above two conditions can be done in polynomial time, but it is not clear how to check this in polynomial time for the amendment procedure. Throughout the rest of this section, we assume that a NECESSARY WINNER instance $(\mathcal{P}:=$ $\left.\left(A, V,\left(\succ_{1}, \succ_{2}, \cdots, \succ_{n}\right)\right), p, \mathcal{B}\right)$ is given.

\subsubsection{Successive Procedure}

We start with the successive procedure and show that deciding whether our preferred alternative is not necessarily a successive winner can be solved in polynomial time. We introduce some notions regarding the alternatives in a (possibly not linear) agenda.

Definition $6\left(\mathcal{B}_{c}^{\leftarrow}, \mathcal{B}_{c}^{\|}\right.$, and $\left.\mathcal{B}_{c}^{\Rightarrow}\right)$. Given a partial agenda $\mathcal{B}$ (which is possibly not linear), for each alternative $c \in A$, let $\mathcal{B}_{c}^{\leftarrow}$ be the set of all alternatives $c^{\prime}$ that are ordered in front of $c$ by $\mathcal{B}$ (that is, $\mathcal{B}: c^{\prime} \triangleright c$ ). Let $\mathcal{B}_{c}^{\|}$be the set of all alternatives $c^{\prime}$ whose relative positions to $c$ are not specified by $\mathcal{B}$ (that is, $\mathcal{B}: c \|_{\triangleright} c^{\prime}$ ), and let $\mathcal{B}_{c}^{\Rightarrow}$ be the set of all alternatives $c^{\prime}$ that are ordered behind $c$ by $\mathcal{B}$ (that is, $\mathcal{B}: c \triangleright c^{\prime}$ ).

Note that for each alternative $c \in A$, the three sets $\mathcal{B}_{c}^{\leftarrow}, \mathcal{B}_{c}^{\|}$, and $\mathcal{B}_{c}^{\Rightarrow}$ are pairwise disjoint and that $\mathcal{B}_{c}^{\leftarrow} \cup \dot{\mathcal{B}} \mathcal{B}_{c}^{\|} \dot{\cup} \mathcal{B}_{c}^{\Rightarrow}=A \backslash\{c\}$.

We derive the main idea behind our polynomial-time algorithm from the following simple observation.

Observation 7. Alternative $p$ is not a necessary successive winner if and only if there is a completion $\left(\mathcal{P}^{*}, \mathcal{L}\right)$ of profile $\mathcal{P}$ and agenda $\mathcal{B}$ such that some other alternative may win, that is, such that

1. $p$ is not an $\left(\mathcal{L}_{p}^{\Rightarrow} \cup\{p\}\right)$-majority winner, or

2. $\mathcal{L}_{p}^{\Leftarrow}$ contains an alternative $c$ that is an $\left(\mathcal{L}_{c}^{\Rightarrow} \cup\{c\}\right)$-majority winner.

We will show that checking whether there is a completion satisfying one of the above conditions can be done in polynomial time. First, we need some more notions and we need to reformulate the above conditions.

Definition 7 (c-discriminating and $c$-privileging). Let $c$ be an arbitrary alternative. Consider a specific linear preference order $\succ^{*}$ that completes a partial preference order $\succ$ such that for each incomparable pair $X$ of alternatives in $\succ$ with $c \notin X$, the relative order of this pair $X$ in $\succ^{*}$ is determined by an arbitrary but fixed linear order. We say that this specific preference order $\succ^{*}$ is $c$-discriminating if for each alternative $c^{\prime}$ that is incomparable to $c$ in $\succ$, it holds that $c^{\prime} \succ^{*} c$. Similarly, $\succ^{*}$ is $c$-privileging if for each alternative $c^{\prime}$ that is incomparable to $c$ in $\succ$, it holds that $c \succ^{*} c^{\prime}$.

Now, consider a profile $\mathcal{P}^{*}$ that completes $\mathcal{P}$. We say that $\mathcal{P}^{*}$ is $c$-discriminating if the preference order of each voter in $\mathcal{P}^{*}$ is $c$-discriminating and that $\mathcal{P}^{*}$ is c-privileging if the preference order of each voter in $\mathcal{P}^{*}$ is $c$-privileging.

Note that a $c$-discriminating (resp. $c$-privileging) profile is unique. We give an example to illustrate these two concepts. 
Example 5. Let $\mathcal{P}$ be a preference profile with four alternatives $a, b, c, d$, and two voters $v_{1}, v_{2}$ whose partial preference orders are specified as

$$
v_{1}: b \succ c \succ d \text { and } v_{2}: d \succ b .
$$

Consider the fixed order $a \succ b \succ c \succ d$ over the alternatives.

The profile with the following preference orders

$$
v_{1}: a \succ b \succ c \succ d \text { and } v_{2}: a \succ d \succ b \succ c \text {, }
$$

is $c$-discriminating; note that it is unique with respect to the fixed order.

The profile with the following preference orders

$$
v_{1}: b \succ c \succ a \succ d \text { and } v_{2}: c \succ a \succ d \succ b \text {, }
$$

is $c$-privileging; note that it is unique with respect to the fixed order.

Now, we can rephrase Conditions 1 and 2 from Observation 7 so that we can verify them in polynomial time.

Lemma 3. There is a completion $\left(\mathcal{P}^{*}, \mathcal{L}\right)$ of $(\mathcal{P}, \mathcal{B})$ satisfying Condition 1 from Observation 7 if and only if $p$ is not an $\left(A \backslash \mathcal{B}_{p}^{\leftarrow}\right)$-majority winner in the p-discriminating completion of $\mathcal{P}$.

Proof. For the "only if" case, suppose that $\left(\mathcal{P}^{*}, \mathcal{L}\right)$ completes $(\mathcal{P}, \mathcal{B})$ and satisfies Condition 1. This means that at least half of the voters in $\mathcal{P}^{*}$ prefer some alternative $c \in \mathcal{L}_{p}^{\Rightarrow}$ to $p$. Since $\mathcal{L}$ completes $\mathcal{B}$, it follows that $\mathcal{L}_{p}^{\Rightarrow} \subseteq A \backslash\left(\mathcal{B}_{p}^{\leftarrow} \cup\{p\}\right)$. Let $\mathcal{P}^{* *}$ be the $p$-discriminating profile. Since each voter in $\mathcal{P}^{*}$ who prefers some alternative $c \in \mathcal{L}_{p}^{\Rightarrow}$ to $p$ will certainly still prefer $c$ to $p$ in $\mathcal{P}^{* *}$ (because this profile "discriminates" $p$ ), it must hold that at least half of the voters in $\mathcal{P}^{* *}$ prefer some alternative $c \in \mathcal{L}_{p}^{\Rightarrow} \subseteq A \backslash\left(\mathcal{B}_{p}^{\Leftarrow} \cup\{p\}\right)$ to $p$. Thus, $p$ is not an $\left(A \backslash \mathcal{B}_{p}^{\Rightarrow}\right)$-majority winner in $\mathcal{P}^{* *}$.

For the "if" case, suppose that $p$ is not an $\left(A \backslash \mathcal{B}_{p}^{\Leftarrow}\right)$-majority winner in the $p$-discriminating profile $\mathcal{P}^{* *}$. Consider an arbitrary agenda $\mathcal{L}$ that satisfies

$$
\mathcal{B}_{p}^{\Leftarrow} \triangleright p \triangleright\left(\mathcal{B}_{p}^{\|} \cup \mathcal{B}_{p}^{\Rightarrow}\right) .
$$

One can verify that $\left(\mathcal{P}^{* *}, \mathcal{L}\right)$ satisfies Condition 1 because $p$ is not a $\left(\mathcal{B}_{p}^{\|} \cup \mathcal{B}_{p}^{\Rightarrow} \cup\{p\}\right)$-majority winner.

Lemma 4. Assume that no completion of $(\mathcal{P}, \mathcal{B})$ satisfies Condition 1 from Observation 7. Then, there is a completion $\left(\mathcal{P}^{*}, \mathcal{L}\right)$ of $(\mathcal{P}, \mathcal{B})$ satisfying Condition 2 from Observation 7 if and only if there is some alternative $b \in \mathcal{B}_{p}^{\Leftarrow}$ being a $\left(\mathcal{B}_{b}^{\Rightarrow} \cup\{b\}\right)$-majority winner in the $b$-privileging completion of $\mathcal{P}$.

Proof. For the "only if" case, suppose that $\left(\mathcal{P}^{*}, \mathcal{L}\right)$ is a completion of $(\mathcal{P}, \mathcal{B})$ which satisfies Condition 2 and let $c \in \mathcal{L}_{p}^{\Leftarrow}$ be such an $\left(\mathcal{L}_{c}^{\Rightarrow} \cup\{c\}\right)$-majority winner. This implies that $c$ beats $p$ in $\mathcal{P}^{*}$ because $p \in \mathcal{L}_{c}^{\Rightarrow}$. Observe that $c \in \mathcal{B}_{p}^{\leftarrow} \cup \mathcal{B}_{p}^{\|}$holds because $\mathcal{L}$ completes $\mathcal{B}$. If $c \in \mathcal{B}_{p}^{\|}$holds, that is, if $\mathcal{B}$ does not specify the relative order of $c$ and $p$, then the same 


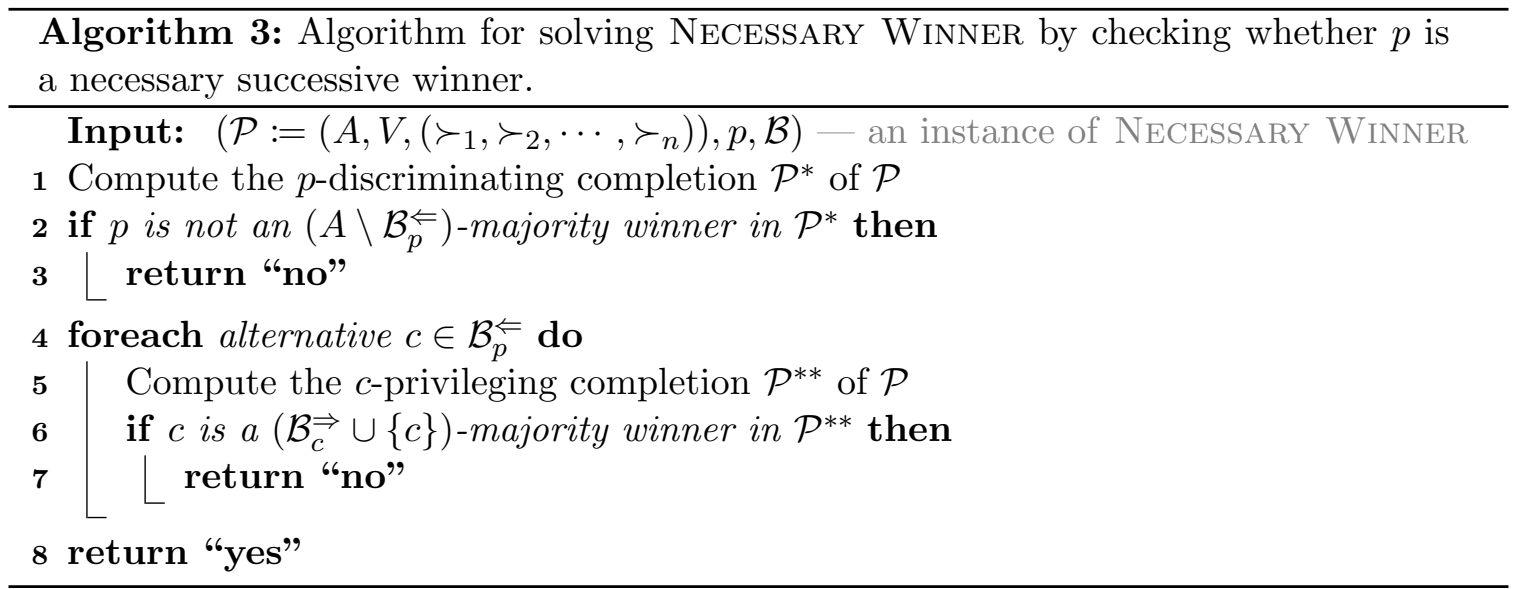

completing profile $\mathcal{P}^{*}$ and a completing agenda of $\mathcal{B}$ with $p \triangleright c$ satisfy Condition 1 which is not possible by our assumption. Thus, we have that $c \in \mathcal{B}_{p}^{\leftarrow}$.

Now, we show that $c$ is a $\left(\mathcal{B}_{c}^{\Rightarrow} \cup\{c\}\right)$-majority winner in the $c$-privileging completion $\mathcal{P}^{* *}$ of $\mathcal{P}$. Observe that each voter $v$ in $\mathcal{P}^{*}$ who prefers $c$ to $\mathcal{L}_{c}^{\Rightarrow}$ must also prefer $c$ to $\mathcal{B}_{c}^{\Rightarrow}$ because $\mathcal{L}$ completes $\mathcal{B}$. Together with the assumption that $c$ is an $\left(\mathcal{L}_{c}^{\Rightarrow} \cup\{c\}\right)$-majority winner in $\mathcal{P}^{*}$, this implies that more than half of the voters in $\mathcal{P}^{*}$ prefer $c$ to $\mathcal{B}_{c}^{\Rightarrow}$. Since each voter in $\mathcal{P}^{*}$ who prefers $c$ to $\mathcal{B}_{c}^{\Rightarrow}$ will still prefer $c$ to $\mathcal{B}_{c}^{\Rightarrow}$ in the $c$-privileging profile $\mathcal{P}^{* *}$, more than half of the voters in $\mathcal{P}^{* *}$ prefer $c$ to $\mathcal{B}_{c}^{\Rightarrow}$.

For the "if" case, suppose that $\mathcal{B}_{p}^{\Leftarrow}$ contains an alternative $b$ that is a $\mathcal{B}_{b} \cup\{b\}$-majority winner in the $b$-privileging completion $\mathcal{P}^{* *}$ of $\mathcal{P}$. Now, consider a completing agenda $\mathcal{L}$ that satisfies

$$
\mathcal{B}_{b}^{\leftarrow} \triangleright \mathcal{B}_{b}^{\|} \triangleright c \triangleright \mathcal{B}_{b}^{\Rightarrow}
$$

We can easily verify that $\left(\mathcal{P}^{* *}, \mathcal{L}\right)$ satisfies Condition 2 (with respect to $b$ ).

Now, we have all ingredients to show that deciding on a necessary successive winner is polynomial-time solvable.

Theorem 8. For the successive procedure, NECESSARY Winner can be solved in $O\left(n \cdot m^{3}\right)$ time.

Proof. By Observation 7 and by Lemmas 3 and 4, we can conclude that $p$ is not a necessary winner if and only if

1. $p$ is not an $\left(A \backslash \mathcal{B}_{p}^{\leftarrow}\right)$-majority winner in the $p$-discriminating completion of $\mathcal{P}$ or

2. $\mathcal{B}_{p}^{\Leftarrow}$ contains an alternative $c$ which is a $\left(\mathcal{B}_{c}^{\Rightarrow} \cup\{c\}\right)$-majority winner in the $c$-privileging completion of $\mathcal{P}$.

With regard to the second requirement, we remark that a $\left(\mathcal{B}_{c}^{\Rightarrow} \cup\{c\}\right)$-majority winner is not guaranteed to win under the successive procedure because some other alternative $c^{\prime}$ from $\mathcal{B}_{c}^{\leftarrow}$ could have been already a $\left(\mathcal{B}_{c^{\prime}}^{\Rightarrow} \cup\left\{c^{\prime}\right\}\right)$-majority winner. Nevertheless, both cases imply that $p$ will not be a successive winner. 
Algorithm 3 checks whether one of the two requirements is fulfilled. Fortunately, this can be done in polynomial time: Computing the $p$-discriminating or the $c$-privileging completion for some alternative $c \in A \backslash\{p\}$ takes $O\left(n \cdot m^{2}\right)$ time and finding the majority winner also takes $O\left(n \cdot m^{2}\right)$ time. The algorithm iterates at most $m$ times through the loop in Steps (4)(7). Altogether it takes $O\left(n \cdot m^{3}\right)$ time.

\subsubsection{Amendment PRocedure}

Adapting the VERTEx COVER reduction from the proof of Theorem 6, we can show that NeCESSARY WinNer for the amendment procedure is coNP-hard.

Theorem 9. For the amendment procedure, NECESSARY WINNER with the given agenda being linear is coNP-complete.

Proof. Recall that in the proof of Theorem 6 we constructed a profile $\mathcal{P}:=(A, V)$ with $2 r-1$ voters and a linear agenda $\mathcal{B}$ for a given instance $(G=(U, E), h)$ of VerTEx Cover, and we showed that $G$ admits no vertex cover of size at most $h$ if and only if our preferred alternative $p$ is not a possible amendment winner. Note that $p$ is not a possible winner if and only if in some completion of the profile $\mathcal{P}$,

1. the helper alternative $b$ beats the dummy alternative $d$ or

2. at least one edge alternative $c_{\ell}$ beats $p$.

Since all $(r-1)$ auxiliary voters and at least one vertex voter prefer each edge alternative to alternative $b$, each edge alternative beats $b$. This implies that if $b$ beats $d$ in the second round, then $c_{s}$ beats $b$, and $b$ will be deleted in the fourth round (note that $b$ beats $p$ in all cases). Thus, $p$ is not a possible winner if and only if there is a completion of the profile, where some edge alternative $c_{\ell}, 1 \leq \ell \leq s$, becomes an $(s-\ell+4)^{\text {th }}$-round amendment winner. Since every voter has the same preference order $c_{1} \succ c_{2} \succ \cdots \succ c_{s}$ over all edge alternatives, edge alternative $c_{1}$ beats every remaining edge alternative $c_{j}, j>1$, and becomes the amendment winner. This implies that $c_{1}$ necessarily wins if and only if $p$ does not possibly win. Hence, the construction in the proof of Theorem 6 provides a polynomialtime reduction from the coNP-complete Co-VERTEX COVER problem to our NeCESSARY WINNER problem for the amendment procedure.

Using the ILP formulation for Possible WinneR under the amendment procedure (Theorem 7), we can check whether there is a possible winner different from $p$. Since $p$ is a

necessary winner if and only if there is no other possible winner, using the results of Lenstra (1983), Kannan (1987), and Frank and Tardos (1987) we can conclude the following.

Corollary 3. Let $m$ denote the number of alternatives and $n$ denote the number of voters of a given NECESSARY WinNER instance. Then, for the amendment procedure, NECESSARY WinNer can be solved in $O\left(\rho^{2.5 \rho+o(\rho)+2} \cdot \log (n+2)\right)$ time, where $\rho=m ! \cdot 2^{m^{2}}$.

\subsection{The Case of Weighted Voters}

If each voter comes with a weight, then for the amendment procedure, PossiBLE WinneR and NeCESSARY Winner are already intractable when the number of alternatives is three 
and four, respectively (Pini et al., 2011; Lang et al., 2012). For the successive procedure, we also obtain weak NP-hardness for Weighted Possible Winner.

Theorem 10. For the successive procedure, Weighted Possible Winner is weakly NPhard even for three alternatives and when the agenda $\mathcal{B}$ is linear.

Proof. We show NP-hardness by providing a polynomial-time reduction from the weakly NP-complete PARTition problem (Garey \& Johnson, 1979).

\section{PARTITION}

Input: A multi-set $X:=\left(x_{1}, x_{2}, \ldots, x_{r}\right)$ of positive integers.

Question: Is there a perfect partition $X_{1} \dot{\cup} X_{2}=X$ of the integers such that both parts sum up to the same value, that is, $\sum_{x \in X_{1}} x=\sum_{x \in X_{2}} x$ ?

Let $X:=\left(x_{1}, x_{2}, \ldots, x_{r}\right)$ be a PARTition instance with $\sum_{x \in X} x=2 K$. We construct a Weighted Possible Winner instance $\left(\left(A, V,\left(\succ_{1}, \succ_{2}, \cdots, \succ_{n}\right)\right), p, \mathcal{B}, \omega: V \rightarrow \mathbb{N}\right)$ with $\omega$ being the weight function. The set $A$ of alternatives consists of the preferred alternative $p$ and two further alternatives $a$ and $b$ :

$$
A:=\{p, a, b\} .
$$

The set $V$ of voters consists of $r$ number voters and one dummy voter:

1. For each integer $x_{i} \in X$ (which is positive), construct one number voter $v_{i}$ with a partial order specified by $a \succ p$ and with weight $\omega\left(v_{i}\right):=x_{i}$.

2. Construct one additional dummy voter $d$ with linear order $p \succ b \succ a$ and with weight $\omega(d):=1$.

Finally, the partial agenda $\mathcal{B}$ is linear and set to $a \triangleright b \triangleright p$. This completes the construction, which clearly can be computed in polynomial time.

Before going into the details of the proof, observe that the total weight of all voters is $2 K+1$ and, hence, the majority quota is $K+1$.

For the "only if" part, assume that there is a perfect partition $X_{1} \cup X_{2}=X$ of the integers such that $\sum_{x \in X_{1}} x=\sum_{x \in X_{2}} x=K$. Then, we complete the profile as follows. For each number voter $v_{i}$, if $x_{i} \in X_{1}$, then the preference order of voter $v_{i}$ is completed to $a \succ p \succ b$; otherwise, the preference order of voter $v_{i}$ is completed to $b \succ a \succ p$. By our agenda $\mathcal{B}$, alternative $a$ will be deleted in the second round because it is not a majority winner (only the voters corresponding to the integers in $X_{1}$ prefer $a$ to $\{p, b\}$ ). In the profile restricted to $b$ and $p$, the dummy voter plus the voters that correspond to the integers in $X_{1}$ prefer $p$ to $b$; the sum of their weights is $K+1$. Thus, $p$ beats $b$ and becomes a winner.

For the "if" part, assume that there is a completion $\mathcal{P}^{*}$ of the profile where $p$ is a successive winner. This implies that $a$ is not a majority winner. Thus, by the preference order of the dummy voter which has weight one, the sum of the weights of the number voters that have preference order $b \succ a \succ p$ is at least $K$. In the third round, $p$ must beat $b$, which implies that the sum of the weights of the number voters that have preference order $b \succ a \succ p$ is at most $K$. Summarizing, the number voters that have preference order $b \succ a \succ p$ have a total weight equal to $K$. The corresponding integers sum up to $K$. 
As already mentioned in the beginning of Section 6.3, Pini et al. (2011) and Lang et al. (2012) showed that Weighted Necessary Winner is weakly NP-hard for the amendment procedure, even for four alternatives. We complement this result by showing that it is polynomial-time solvable for the successive procedure, and it is linear-time solvable for the amendment procedure when the number of alternatives is at most three.

Theorem 11. Let $m$ denote the number of alternatives, $n$ denote the number of voters, and $w$ denote the sum of the weights (encoded in binary) of all voters of a given NECESSARY Winner instance. The following holds. 1. For the successive procedure, WeIGHTED NeCESSARY WinNer can be solved in $O\left(w \cdot n \cdot m^{3}\right)$ time. 2. For the amendment procedure, Weighted Necessary Winner can be solved in linear time for up to three alternatives.

Proof. First, we observe that our algorithm (Theorem 8: Algorithm 3) for checking whether an alternative is a necessary successive winner of a given profile without weights can be easily adapted to also solve the case of weighted voters. Since comparing the weights of the voters can be done in $w$ time, where $w$ is the total sum of the weights encoded in binary, we obtain that for the successive procedure, WeIghted NECESSARY WinNer can be solved in $O\left(w \cdot n \cdot m^{3}\right)$ time.

We show that for up to three alternatives, Weighted NeCEssary Winner is lineartime solvable. This is closely related to computing possible and necessary Condorcet winners. To show our result, for each two alternatives $a$ and $b$, let $\omega(a, b)$ be the sum of the weights of all voters who prefer $a$ to $b$.

We check all (up to) six completions $\mathcal{L}$ of a given partial agenda $\mathcal{B}$ and answer that our preferred alternative $p$ is a necessary amendment winner only if all checks return "yes". First, if $p$ is in one of the first two positions in agenda $\mathcal{L}$, then the problem is equivalent to asking whether $p$ is a necessary Condorcet winner, that is, whether all completions of the preference orders make $p$ beat the other(s), one by one. The answer to the latter is yes if and only if for each remaining alternative $c$, the sum of the weights of all voters who prefer $p$ to $c$ is more than half of the total sum of weights. This can be checked in linear time.

We are left with the very last case where the profile has three alternatives, denoted by $a, b$, and $p$, and where the preferred alternative $p$ is in the last position in the agenda $\mathcal{L}$. Let $K$ be the total sum of weights. In this case, $p$ is a necessary winner if and only if either $p$ is a Condorcet winner or the sum of the weights of all voters who prefer $p$ to the necessary Condorcet winner among $a$ and $b$ is greater than $K / 2$ :

(1) If $\omega(p, a)>K / 2$ and if $\omega(p, b)>K / 2$, then answer "yes";

(2) Otherwise, if $\omega(p, a)>K / 2$ and if $\omega(a, b)>K / 2$, then answer "yes";

(3) Otherwise, if $\omega(p, b)>K / 2$ and if $\omega(b, a)>K / 2$, then answer "yes";

(4) Otherwise, answer "no".

The condition stated in Step (1) implies that $p$ is a Condorcet winner. As we know, if a Condorcet winner exists, then it is the only unique amendment winner (Observation 1). Thus, Step (1) gives a correct answer. If the conditions in Step (1) does not hold, then the first inequality in Step (2) implies $\omega(p, b) \leq K / 2$. The second inequality in Step (2) implies 
that in every completion of the profile, $a$ will beat $b$. In this case, $p$ will beat $a$ and becomes an amendment winner. Thus, Step (2) gives a correct answer. Analogously, we can show that Step (3) also gives a correct answer.

If neither of the first three steps applies, then we obtain that one of the conditions between $\omega(p, a) \leq K / 2$ and $\omega(p, b) \leq K / 2$ and one of the conditions between $\omega(a, b) \leq K / 2$ and $\omega(b, a) \leq K / 2$ must hold. This results in four possible combinations of the conditions, each of which ensures that $p$ is not a necessary winner. Thus, the last step also gives a correct answer. All checks can be done in linear time.

\section{Conclusion}

Our work indicates that, from a computational perspective, the amendment procedure seems superior to the successive procedure against agenda control and strategic voting. Our work suggests that most European and Latin parliaments should rather go the Anglo-American way, that is, they should use the amendment procedure instead of the successive procedure.

We see this paper as an initial step towards computational complexity studies of parliamentary voting procedures. After identifying the computational complexity of several voting problems for both studied procedures, following the spirit of Betzler et al. (2009), it would be of interest to complement our computational hardness results for possible and necessary winner problems with a refined complexity analysis concerning tractable special cases. For instance, our NP-hardness reductions for the possible winner problems assume that voters may have arbitrary partial preferences. It would be interesting to know whether this still holds if voter preferences have a certain structure, such as, for instance, being single-peaked (Black, 1958) or semi-single-peaked (Rasch, 1987).

For both procedures, we obtain that our NP-complete POssible Winner problems are fixed-parameter tractable for the parameter "number $m$ of alternatives". With respect to the parameter "number $n$ of voters", however, we could not settle their parameterized complexity.

It would be natural to also adopt a more game-theoretic view on the strategic voting scenarios where all voters act strategically, as opposed to CoALitional MANipulation, where only the voters of a coalition vote strategically (with the same goal) and all other voters vote sincerely. Note that we can see both the successive procedure and the amendment procedure as repeated games, so a natural concept to consider is the subgame-perfect Nash equilibrium (Osborne, 2004).

Finally, it would be also interesting to study further manipulation scenarios for parliamentary voting procedures including, for example, the "sophisticated voting setting", which has been widely studied in the political science literature (see Miller, 1977; Enelow \& Koehler, 1980; McKelvey \& Niemi, 1978; Shepsle \& Weingast, 1982; Banks, 1985; Moulin, 1986; Ordeshook \& Schwartz, 1987), or control by changing the set of alternatives as discussed by Rasch (2014). 


\section{Acknowledgments}

The authors want to thank the anonymous reviewers from IJCAI 2015 and JAIR for improving the paper. An extended abstract of this work has been presented at the 24th International Joint Conference on Artificial Intelligence (IJCAI 2015), Buenos Aires, July, 2015 (Bredereck, Chen, Niedermeier, \& Walsh, 2015). In this long version, we provide two additional fixed-parameter tractability results (Theorem 7 and Corollary 2) and all proofs that were omitted in the conference version.

Robert Bredereck was supported by the German Research Foundation (DFG), research project PAWS, NI 369/10, and by the DFG fellowship BR 5207/2. Main work was done while Jiehua Chen was with TU Berlin. Toby Walsh was supported by the Alexander von Humboldt Foundation while visiting TU Berlin. He is also affiliated with NICTA and the University of New South Wales, Australia. NICTA is funded by the Australian Government through the Department of Communications and the Australian Research Council through the ICT Centre of Excellence Program.

\section{References}

Apesteguia, J., Ballester, M. A., \& Masatlioglu, Y. (2014). A foundation for strategic agenda voting. Games and Economic Behavior, 87, 91-99.

Aziz, H., Brill, M., Fischer, F. A., Harrenstein, P., Lang, J., \& Seedig, H. G. (2015). Possible and necessary winners of partial tournaments. Journal of Artificial Intelligence Research, 54, 493-534.

Banks, J. S. (1985). Sophisticated voting outcomes and agenda control. Social Choice and Welfare, 1(4), 295-306.

Barberà, S., \& Gerber, A. (2017). Sequential voting and agenda manipulation. Theoretical Economics, 12, 211-247.

Bartholdi III, J., Tovey, C., \& Trick, M. (1992). How hard is it to control an election?. Mathematical and Computer Modeling, 16(8/9), 27-40.

Bartholdi III, J. J., \& Orlin, J. B. (1991). Single transferable vote resists strategic voting. Social Choice and Welfare, 8(4), 341-354.

Bartholdi III, J. J., Tovey, C. A., \& Trick, M. A. (1989). The computational difficulty of manipulating an election. Social Choice and Welfare, 6(3), 227-241.

Baumeister, D., \& Rothe, J. (2012). Taking the final step to a full dichotomy of the possible winner problem in pure scoring rules. Information Processing Letters, 112(5), 186190.

Betzler, N., \& Dorn, B. (2010). Towards a dichotomy of finding possible winners in elections based on scoring rules. Journal of Computer and System Sciences, 76 (8), 812-836.

Betzler, N., Hemmann, S., \& Niedermeier, R. (2009). A multivariate complexity analysis of determining possible winners given incomplete votes. In Proceedings of the 21st International Joint Conference on Artificial Intelligence (IJCAI'09), pp. 53-58. AAAI Press. 
Black, D. (1958). The Theory of Committees and Elections. Cambridge University Press.

Brandt, F., Conitzer, V., Endriss, U., Lang, J., \& Procaccia, A. (Eds.). (2016). Handbook of Computational Social Choice. Cambridge University Press.

Bredereck, R., Chen, J., Faliszewski, P., Guo, J., Niedermeier, R., \& Woeginger, G. J. (2014). Parameterized algorithmics for computational social choice: Nine research challenges. Tsinghua Science and Technology, 19(4), 358-373.

Bredereck, R., Chen, J., Niedermeier, R., \& Walsh, T. (2015). Parliamentary voting procedures: Agenda control, manipulation, and uncertainty. In Proceedings of the 24rd International Joint Conference on Artificial Intelligence (IJCAI '15), pp. 164-170. AAAI Press.

Conitzer, V., Sandholm, T., \& Lang, J. (2007). When are elections with few candidates hard to manipulate? Journal of the ACM, 54(3), 1-33.

Cormen, T. H., Leiserson, C. E., Rivest, R. L., \& Stein, C. (2009). Introduction to Algorithms. MIT Press.

Enelow, J. M., \& Koehler, D. H. (1980). The amendment in legislative strategy: Sophisticated voting in the U.S. Congress. The Journal of Politics, 42(2), 396-413.

Faliszewski, P., Hemaspaandra, E., \& Hemaspaandra, L. (2010). Using complexity to protect elections. Communications of the ACM, 53(11), 74-82.

Faliszewski, P., \& Rothe, J. (2016). Control and bribery in voting. In Brandt, F., Conitzer, V., Endriss, U., Lang, J., \& Procaccia, A. D. (Eds.), Handbook of Computational Social Choice, chap. 7. Cambridge University Press.

Farquharson, R. (1969). Theory of Voting. Yale University Press.

Frank, A., \& Tardos, É. (1987). An application of simultaneous Diophantine approximation in combinatorial optimization. Combinatorica, 7(1), 49-65.

Garey, M. R., \& Johnson, D. S. (1979). Computers and Intractability-A Guide to the Theory of NP-Completeness. W. H. Freeman and Company.

Good, I. J. (1971). A note on Condorcet sets. Public Choice, 10(1), 97-101.

GovTrack (2011-2012). GovTrack: Website for tracking the United States Cxongress.. Retrieved from http://www.govtrack.us/ for years 2011-2012.

Harary, F., \& Moser, L. (1966). The theory of round robin tournaments. The American Mathematical Monthly, 73(3), 231-246.

Hazon, N., Aumann, Y., Kraus, S., \& Wooldridge, M. (2012). On the evaluation of election outcomes under uncertainty. Artificial Intelligence, 189, 1-18.

Jung, J. P. (1989). Condorcet consistent binary agendas under incomplete information. In Models of Strategic Choice in Politics. University of Michigan Press.

Kannan, R. (1987). Minkowski's convex body theorem and integer programming. Mathematics of Operations Research, 12(3), 415-440.

Konczak, K., \& Lang, J. (2005). Voting procedures with incomplete preferences. In Proceedings of IJCAI-05 Multidisciplinary Workshop on Advances in Preference Handling, pp. $124-129$. 
Lang, J., Pini, M. S., Rossi, F., Salvagnin, D., Venable, K. B., \& Walsh, T. (2012). Winner determination in voting trees with incomplete preferences and weighted votes. Autonomous Agents and Multi-Agent Systems, 25(1), 130-157.

Lenstra, H. W. (1983). Integer programming with a fixed number of variables. Mathematics of Operations Research, 8(4), 538-548.

Mattei, N., \& Walsh, T. (2013). Preflib: A library for preferences http://www.preflib.org. In Proceedings of the 3rd International Conference on Algorithmic Decision Theory (ADT '13), Vol. 8176 of Lecture Notes in Computer Science, pp. 259-270. Springer. http://preflib.org.

McKelvey, R. D., \& Niemi, R. G. (1978). A multistage game representation of sophisticated voting for binary procedures. Journal of Economic Theory, 18(1), 1-22.

Miller, N. R. (1977). Graph-theoretical approaches to the theory of voting. American Journal of Political Science, 21(4), 769-803.

Moulin, H. (1986). Choosing from a tournament. Social Choice and Welfare, 3(4), 271-291.

Nurmi, H. (2005). Voting Procedures under Uncertainty. Springer.

Ordeshook, P. C., \& Palfrey, T. R. (1988). Agendas, strategic voting, and signaling with incomplete information. American Journal of Political Science, 32(2), 441-466.

Ordeshook, P. C., \& Schwartz, T. (1987). Agendas and the control of political outcomes. The American Political Science Review, 81(1), 179-200.

Osborne, M. J. (2004). An Introduction to Game Theory. Oxford University Press.

Pini, M. S., Rossi, F., Venable, K. B., \& Walsh, T. (2011). Incompleteness and incomparability in preference aggregation: Complexity results. Artificial Intelligence, 175(7-8), $1272-1289$.

Rasch, B. E. (1987). Manipulation and strategic voting in the Norwegian parliament. Public Choice, 52(1), 57-73.

Rasch, B. E. (2000). Parliamentary floor voting procedures and agenda setting in Europe. Legislative Studies Quarterly, 25(1), 3-23.

Rasch, B. E. (2014). Insincere voting under the successive procedure. Public Choice, 158(34), 499-511.

Rothe, J. (Ed.). (2015). Economics and Computation. Springer.

Rothe, J., \& Schend, L. (2013). Challenges to complexity shields that are supposed to protect elections against manipulation and control: a survey. Annals of Mathematics and Artificial Intelligence, 68(1-3), 161-193.

Schwartz, T. (1972). Rationality and the myth of the maximum. Nô̂s, 6(2), 97-117.

Shepsle, K. A., \& Weingast, B. R. (1982). Uncovered sets and sophisticated voting outcomes with implications for agenda institutions. American Journal of Political Science, 28(1), 49-74.

Smith, J. H. (1973). Aggregation of preferences with variable electorate. Econometrica, $41(6), 1027-1041$. 
Parliamentary Voting Procedures: Agenda Control, Manipulation, \& Uncertainty

Walsh, T. (2007). Uncertainty in preference elicitation and aggregation. In Proceedings of the 22nd Conference on Artificial Intelligence (AAAI '07), pp. 3-8. AAAI Press.

Xia, L., \& Conitzer, V. (2011). Determining possible and necessary winners under common voting rules given partial orders. Journal of Artificial Intelligence Research, 41, 25-67. 